\title{
LATTICE AND HEEGAARD FLOER HOMOLOGIES OF ALGEBRAIC LINKS
}

\author{
EUGENE GORSKY AND ANDRÁS NÉMETHI
}

\begin{abstract}
We compute the Heegaard Floer link homology of algebraic links in terms of the multivariate Hilbert function of the corresponding plane curve singularities. The main result of the paper identifies four homologies: (a) the Heegaard Floer link homology of the local embedded link, (b) the lattice homology associated with the Hilbert function, (c) the homologies of the projectivized complements of local hyperplane arrangements cut out from the local algebra, and (d) a generalized version of the Orlik-Solomon algebra of these local arrangements. In particular, the Poincaré polynomials of all these homology groups are the same, and we also show that they agree with the coefficients of the motivic Poincaré series of the singularity.
\end{abstract}

\section{INTRODUCTION}

Complex analytic/algebraic plane curve singularities provide interesting connections between analytic theory of singularities and low dimensional topology, in particular, knot theory. The rigidity properties of algebraic links help to compute the topological invariants via analytic methods, while knot theory provides topological characterizations for certain analytic invariants (see e.g. [1, 9, 16] and references therein). E.g., in [4] Campillo, Delgado and Gusein-Zade related the multi-variable Alexander polynomial of an algebraic link to the multi-dimensional semigroup of the divisors of analytic functions. They also identified the coefficients of the Alexander polynomial with the Euler characteristics of certain projectivized hyperplane arrangement complements associated with the ring of functions. In this paper, we prove a "homological lift" of their theorem by identifying the Heegaard Floer link homology of the local analytic link with the homology of these hyperplane arrangements, and providing a concrete and computable description of them in terms of classical singularity invariants of algebraic links (Hilbert function, or Alexander polynomial).

Usually, the identification of the Heegaard Floer link homology $H F L^{-}$is very hard, and very few concrete examples are known. For $L$-space links we propose a strategy, which makes a conceptual simplification, however at this generality this strategy is also obstructed seriously at several points. The strategy provides a spectral sequence converging to $H F L^{-}$, whose $E_{2}$ term is a lattice cohomology associated with certain weights, which are determined by the Alexander polynomial. But for a general $L$-space link the collapse of the spectral sequence is not guaranteed.

However, for algebraic links we eliminate all these obstructions as follows. Firstly, in [11] we proved that algebraic links are $L$-space links, hence the strategy runs. Then, we identify the $H F L$-weights needed for the $E_{2}$ (lattice cohomology) term with the values of the Hilbert function of the local algebra (where the multi-filtration is given by valuations induced by the normalization). For this we need an 'analytic inversion' formula, which provides the Hilbert function from the Alexander polynomial.

This Hilbert function is the central singularity invariant, it has a rich structure which will be exploited deeply. Based on this, we analyze the properties of the lattice cohomology (defined in [23] in a very general setup) associated with the Hilbert function weights, and we show that it is isomorphic to the cohomology of certain hyperplane arrangements embedded in the 
ring of functions. For this step we need to use and improve the Orlik-Solomon theory of the cohomology of hyperplane arrangement complements. The next step exploits the structure of Orlik-Solomon cohomology rings (determined by the rigid matroid properties of our Hilbert function). We define a bigrading on the Orlik-Solomon complex and prove a vanishing result which guarantees that the cohomology is supported on a line (with respect to this bigrading). This intrinsic structure and vanishing will imply finally the collapse of the above mentioned spectral sequence involving the $H F L^{-}$theory (showing that all the higher differentials endowed with the bigrading are necessarily trivial).

The final picture identifies the ranks of the following four graded homologies:

(a) The Heegaard Floer link homology of the local embedded link of the germ,

(b) The local lattice homology associated with the Hilbert function,

(c) The (simplicial) homologies of the projectivized complements of local hyperplane arrangements cut out from the local algebra by valuations given by the normalizations of irreducible components,

(d) A generalized version of the Orlik-Solomon algebra of these local arrangements.

In particular, the Poincaré polynomials of all these homology groups are the same, and we also show that they agree with the coefficients of the motivic Poincare series of the singularity germ [6, 10, 18]. Since the homologies have no $\mathbb{Z}$-torsion, the corresponding Poincaré polynomials provide the complete description of the corresponding homologies.

It is important to mention that the above isomorphisms are defined separately for each Alexander grading, which belongs to the lattice $\mathbb{Z}^{r}$ (where $r$ is the number of components of a link). For each lattice point $v \in \mathbb{Z}^{r}$ we define a separate topological space $\mathcal{H}(v)$ (which is either empty or a complement to a hyperplane arrangement) and relate its homology to $\mathrm{HFL}^{-}(v)$. This recovers $\mathrm{HFL}^{-}=\bigoplus_{v} \mathrm{HFL}^{-}(v)$ as a $\mathbb{Z}^{r} \oplus \mathbb{Z}$-graded vector space (for a comment regarding coefficients, see Remark 2.1.2). Here the last $\mathbb{Z}$-grading is the homological grading. (All other homologies in the above list (a)-(d) are graded similarly.)

Some of the important structures present in $\mathrm{HFL}^{-}$are not immediately recovered with this approach. In particular, the Heegaard Floer theory defines operators $U_{1}, \ldots, U_{r}$ which act on $\mathrm{HFL}^{-}$and shift the Alexander grading in various directions. It seems plausible that the action of $U_{i}$ is determined by the Hilbert function too, but we do not study this action in the present paper - such a study would require a comparison of spaces $\mathcal{H}(v)$ for different $v$.

In order to realize the above program, we need to recall/improve several properties of Heegaard Floer link homology of $L$-space links in section 2 and of local algebraic curve singularities (e.g. how to invert the Alexander polynomial to the Hilbert function) (section 3), to develop the theory of lattice cohomology (associated with the Hilbert function weights) (section 4), and to adjust and improve the theory of Orlik-Solomon algebras (section 5). Based on all these we finish the main proof in section 6. Finally, in section 7 we explicitly compute the homologies (a)-(d) for the Hopf link, corresponding to the singularity $\{x y=0\}$.

1.1. The next subsections provide more details on the involved invariants and identifications (for the precise definitions and statements see the next sections).

Trough the paper the following notations will be used. The number of link components will be denoted by $r$. Set $K_{0}=\{1, \ldots, r\}$. Let $e_{i}$ denote the $i$-th coordinate vector in $\mathbb{Z}^{r}$. For a subset $K \subset K_{0}$ we write $e_{K}=\sum_{i \in K} e_{i}$ and $e=e_{K_{0}}=\sum e_{i}$. Given $v \in \mathbb{Z}^{r}$, we define $v_{K}=\sum_{i \in K} v_{i} e_{i} .|K|$ denotes the cardinality of $K$. We set a partial order on $\mathbb{Z}^{r}$ by

$$
u \preceq v \Longleftrightarrow u_{i} \leq v_{i} \text { for all } i \text {. }
$$


1.2. The Hilbert series and the related singularity 'package'. Let $(C, 0)=\left(\cup_{i=1}^{r} C_{i}, 0\right)$ be a reduced plane curve singularity at the origin in $\mathbb{C}^{2}$, where $C_{i}$ are the irreducible components. Let $\gamma_{i}:(\mathbb{C}, 0) \rightarrow\left(C_{i}, 0\right)$ be the normalization of the components. We consider $r$ valuations on the $\mathbb{C}$-algebra $\mathcal{O}=\mathcal{O}_{\mathbb{C}^{2}, 0}$ defined by $\mathfrak{v}_{i}(f)=\operatorname{ord}\left(f\left(\gamma_{i}(t)\right)\right)$, and a $\mathbb{Z}^{r}$-indexed filtration

$$
J(v)=\left\{f \in \mathcal{O} \mid \mathfrak{v}_{i}(f) \geq v_{i} \text { for all } i\right\} .
$$

The Hilbert function $h: \mathbb{Z}^{r} \rightarrow \mathbb{Z}$ is defined by $h(v)=\operatorname{dim} \mathcal{O} / J(v)$, while the multivariable Hilbert series by $H(t)=\sum_{v} h(v) t_{1}^{v_{1}} \cdots t_{r}^{v_{r}}$, cf. 3.1.1. It guides most of the classical analytic and topological invariants of the germ. For example, the multivariable Poincaré series satisfies $P(t)=-H(t) \cdot \prod_{i}\left(1-t_{i}^{-1}\right)$. By [4] $P(t)$ is related to the multivariable Alexander polynomial $\Delta(t)$ as follows: $\Delta(t)=P(t)$ if $r>1$, while $\Delta(t)=(1-t) P(t)$ for $r=1$. This shows that $\Delta(t)$ is determined by the Hilbert series $H(t)$. We prove an 'Inversion Theorem' 3.4 .3 providing an explicit way to recover $H(t)$ from $\Delta(t)$. (This explicit formula can be used to define an analogue of $H(t)$ for any non-algebraic link as well; this plays an important role in the study of $L$-space links in Heegaard Floer link theory: it produces the weights of the lattice complex whose lattice cohomology is the $E_{2}$ term of the spectral sequence, cf. Theorem 1.5.1.)

Another objects determined by the valuations are the topological spaces

$$
\mathcal{H}(v):=\left\{f \in \mathcal{O} \mid \mathfrak{v}_{i}(f)=v_{i} \text { for all } i\right\}
$$

and their projectivizations $\mathbb{P} \mathcal{H}(v)$. Although $\mathcal{H}(v)$ and $\mathbb{P} \mathcal{H}(v)$ are infinite-dimensional, they can be projected onto finite-dimensional varieties with affine fibers. Furthermore, $\mathcal{H}(v)=$ $J(v) \backslash \cup_{i} J\left(v+e_{i}\right)$ (where $e_{i}$ are the base vectors), hence $\mathcal{H}(v)$ is either empty or a complement of a central hyperplane arrangement, see section 3.6. It turns out that the Euler characteristic of $\mathbb{P} \mathcal{H}(v)$ is exactly the coefficient $\pi_{v}$ of $t^{v}$ in the Poincare series $P(t)$. Replacing the Euler characteristic $\pi_{v}$ by the Poincaré polynomial $\pi_{v}(q)$ of the homology of $\mathbb{P H}(v)$ (or by the class of $\mathbb{P} \mathcal{H}(v)$ in the Grothendieck ring of algebraic varieties), we obtain the 'motivic Poincaré series' $\mathcal{P}(t ; q)=\sum_{v} \pi_{v}(q) t^{v}[6,10,18]$.

1.3. The Orlik-Solomon theory. To describe the homology of $\mathcal{H}(v)$, we need some facts from the theory of hyperplane arrangements. Let $\left\{H_{1}, \ldots, H_{r}\right\}$ be a collection of hyperplanes in a complex vector space $V$. Brieskorn in [3] proved that the de Rham cohomology of the complement $\mathcal{H}:=V \backslash \cup_{i} H_{i}$ is generated as an algebra by the classes of 1-forms $z_{i}=\frac{d \ell_{i}}{\ell_{i}}$, where $\ell_{i}$ are the defining equations of $H_{i}$. Orlik and Solomon [24] gave an explicit combinatorial description of the ideal of relations between $z_{i}$ in terms of linear dependencies between $\ell_{i}$ (see 5.2). To connect the Orlik-Solomon theory with the $\mathbb{Z}$-module structure of the lattice and $H F L^{-}$cohomologies, we prove the following improvement of their result (see Theorem 5.2.8).

Theorem 1.3.1. Consider the free anticommutative algebra $\mathcal{E}$ generated by $z_{i}$. It is naturally bigraded: a monomial $\wedge_{i \in K} z_{i}$ has bidegree $(|K|, \rho(K))$, where $\rho(K):=\operatorname{dim} V / \cap_{i \in K} H_{i}$.

(a) There is a differential $\partial_{0}$ on $\mathcal{E}$ of bidegree $(-1,0)$ such that $H_{*}\left(\mathcal{E}, \partial_{0}\right) \simeq H_{*}(\mathcal{H})$.

(b) There is a differential $\partial_{U}=\partial_{0}+U \partial_{1}$ on $\mathcal{E}[U]$ such that $H_{*}\left(\mathcal{E}[U], \partial_{U}\right) \simeq H_{*}(\mathbb{P} \mathcal{H})$.

(c) All classes in the homology of $\partial_{U}$ have $U$-degree 0 and can be presented as sums of monomials $\alpha=\wedge_{i \in K} z_{i}$ such that the hyperplanes $H_{i \in K}$ are independent.

Corollary 1.3.2. (a) The homology of $\partial_{0}$ or $\partial_{U}$ inherits a bidegree, and for the nontrivial generators $|\alpha|=\rho(\alpha)$. Therefore, the bidegrees in non-trivial homology elements lie on a line.

(b) The U-action on $H_{*}\left(\mathcal{E}[U], \partial_{U}\right) \simeq H_{*}(\mathbb{P} \mathcal{H})$ is trivial. 
1.4. The lattice homology. This note introduces the lattice homology of $(C, 0)$. Recall that in [23] the lattice homology of a normal surface singularity was introduced via the lattice provided by its resolution graph (or plumbing graph of the link). That invariant created a bridge between the analytic invariants of the surface singularity and several topological invariants (like SeibergWitten invariant and Heegaard Floer homology) of its 3-dimensional link. The goal of the present construction is similar; nevertheless here we rely on the lattice $\mathbb{Z}^{r}$ discussed above, and the needed weight function is provided by the normalization of $C$, namely by the Hilbert function $h(v)$.

In short, the definition for an arbitrary weight function $w: \mathbb{Z}^{r} \rightarrow \mathbb{Z}$ runs as follows. The lattice complex $\mathcal{L}_{w}^{-}$is generated over $\mathbb{Z}[U]$ by cubes $\square$ of all dimensions in $\mathbb{R}^{r}$, with vertices in the lattice $\mathbb{Z}^{r}$. For such a cube we define $w(\square)=\max _{x \in \square \cap \mathbb{Z}^{r}} w(x)$. The differential is defined as

$$
\partial_{U}\left(U^{m} \square\right)=U^{m} \cdot \sum_{i} \varepsilon_{i} U^{w(\square)-w\left(\square_{i}\right)} \square_{i}
$$

where $\square_{i}$ are the oriented boundary cubes of $\square$, and $\varepsilon_{i}$ are the corresponding signs (as in the boundary operator of the classical cubic homology). We define the homological degree of the generators by $\operatorname{deg}\left(U^{m} \square\right)=-2 m+\operatorname{dim}(\square)-2 w(\square) ; \partial_{U}$ decreases it by one.

The complex $\mathcal{L}_{w}^{-}$is naturally $\mathbb{Z}^{r}$-filtered: the subcomplex $\mathcal{L}_{w}^{-}(v)$ is generated by the cubes contained in the positive quadrant originating at $v$. One of our main theorems describes the homology of the subcomplexes $\mathcal{L}_{w}^{-}(v)$ and the associated graded complexes gr $\mathcal{L}_{w}^{-}$for all $v$.

Theorem 1.4.1. $\quad$ (a) If $w$ is non-decreasing (that is, $w(v) \leq w(u)$ for $v \preceq u$ ), then $H^{*}\left(\mathcal{L}_{w}^{-}(v)\right) \simeq$ $\mathbb{Z}[U]$ with a generator of homological degree $-2 w(v)$.

(b) In the algebraic case (that is, if $w=h$ ), the Poincaré polynomial $P_{\mathrm{gr}_{v} \mathcal{L}_{h}^{-}}(t)$ of the homology of $\operatorname{gr}_{v} \mathcal{L}_{h}^{-}$agrees with the $v$-coefficient in the motivic Poincaré series:

$$
P_{\mathrm{gr}_{v} \mathcal{L}_{h}^{-}}\left(-t^{-1}\right)=t^{h(v)} \pi_{v}(t) .
$$

(c) The following (co)homologies are isomorphic:

$$
H_{-2 h(v)-*}\left(\operatorname{gr}_{v} \mathcal{L}_{h}^{-}\right) \simeq H^{*}(\mathbb{P} \mathcal{H}(v))
$$

and both spaces are free $\mathbb{Z}$-modules.

(d) The induced U-action on $H_{*}\left(\operatorname{gr}_{v} \mathcal{L}_{h}^{-}\right)$is trivial.

We prove the parts of this theorem in Theorems 4.1.7, 4.2.1 and 5.3.1.

1.5. Heegaard Floer link homology. We relate the Heegaard Floer link homology HFL ${ }^{-}$of an algebraic link to lattice homology of the corresponding plane curve singularity. (For the definition of $\mathrm{HFL}^{-}$see [27, 28, 29, 30] and [33]).

Recall that an $L$-space is a 3-manifold with minimal possible rank of its Heegaard Floer homology, and an $L$-space link is a link in $S^{3}$ such that a sufficiently large surgery of $S^{3}$ along its components yield an $L$-space. Ozsváth and Szabó proved in [27] that the Heegaard Floer homology of an $L$-space knot is determined by its Alexander polynomial. Hedden proved in [12] than every algebraic knot is an $L$-space knot. As a consequence, Heegaard Floer homology of an algebraic knot is determined by its Alexander polynomial. However, for $L$-space links is not known if their Heegaard Floer link homology is determined by the multivariable Alexander polynomial.

As a generalization of the above facts valid for knots, we propose the following program. First, in [11] (motivated by the present manuscript), the authors observe that all algebraic links are $L$-space links. Then, by the general theory of Ozsváth and Szabó of $L$-space links and 
by 'Large Surgery Theorem' of Manolescu and Ozsváth [15], such a link $L \subset S^{3}$ provides a function $g: \mathbb{Z}^{r} \rightarrow \mathbb{Z}$ as follows (we call it $H F L$-weight function). The $H F L^{-}$complex is a $\mathbb{Z}\left[U_{1}, \ldots, U_{r}\right]$ module with Alexander filtration $\left\{A^{-}(v)\right\}_{v \in \mathbb{Z}^{r}}$, where $U_{i}\left(A^{-}(v)\right) \subset A^{-}\left(v+e_{1}\right)$. $A^{-}(v)$ is a subcomplex and a $\mathbb{Z}\left[U_{1}, \ldots, U_{r}\right]$ submodule. Its homology is a free rank one $\mathbb{Z}[U]-$ module (with $U=U_{1}$ ). Then $g(v)$ is essentially the homological degree of its unique generator (similarly to Theorem 1.4.1 (a)). The function $g(v)$ is determined by the multi-variable Alexander polynomial of $L$ (Theorem 2.2.11). We prove the following (see Theorem 2.2.10).

Theorem 1.5.1. Let $L$ be an $L$-space link and let $g: \mathbb{Z}^{r} \rightarrow \mathbb{Z}$ be its $H F L$-weight function. Then for each fixed $v \in \mathbb{Z}^{r}$ there exists a spectral sequence with the following properties:

(a) The $E^{1}$ page can be identified (as a $\mathbb{Z}[U]$ module) with the lattice complex $\operatorname{gr}_{v} \mathcal{L}_{g}^{-}$ associated with $g(v)$.

(b) The $E^{2}$ page is isomorphic (over $\mathbb{Z}$ ) to the local lattice homology associated with $g(v)$.

(c) The $E^{\infty}$ page is isomorphic (as graded $\mathbb{Z}$-module, where the grading is the homological one) to $\mathrm{HFL}^{-}(L, v)$, the Heegaard Floer link homology of $L$ with Alexander grading $v$. Moreover, the spectral sequence collapses at $E^{r}$ page (or earlier).

(d) If $r \leq 3$ then the spectral sequence collapses at the $E^{2}$ page.

For algebraic links the following additional facts hold (cf. Theorems 6.1.2 and 6.1.3).

Theorem 1.5.2. If $L$ is the link of a plane curve singularity $(C, 0)$ then the $H F L$-weight function $g(v)$ coincides with the Hilbert function $h(v)$. Moreover, the spectral sequence always collapses at the $E^{2}$ page.

Corollary 1.5.3. If $L$ is the algebraic link of $(C, 0)$ then for each fixed $v \in \mathbb{Z}^{r}$ one has

$$
\mathrm{HFL}^{-}(L, v) \simeq H_{*}\left(\operatorname{gr}_{v} \mathcal{L}_{h}^{-}\right) \simeq H^{-2 h(v)-*}(\mathbb{P} \mathcal{H}(v))
$$

(isomorphism of graded $\mathbb{Z}$ modules). Moreover, the Poincaré polynomial of the Heegaard Floer link homology is described by Theorem 1.4.1 by the coefficients of the motivic Poincaré series. Furthermore, $\mathrm{HFL}^{-}(L, v) \neq 0$ if and only if $v$ belongs to the semigroup of $(C, 0)$.

Theorem 1.5.1 can be compared with [31, Theorem 1.1], where a similar spectral sequence from a different form of lattice homology (associated with a plumbing graph) to Heegaard Floer homology was considered. For the first part of Theorem 1.5.2 we use the 'Inversion Theorem' 3.4.3. and in the proof of collapse we use some specific properties of the lattice homology and Orlik-Solomon algebras established in Theorem 4.2.1 (cf. Corollary 1.3.2).

\section{ACKNOWLEDGEMENTS}

The authors are grateful to S. Gusein-Zade, J. Fernández de Bobadilla, M. Hedden, C. Manolescu, J. Moyano-Fernández, P. Ozsváth, J. Rasmussen and Z. Szabó for the useful remarks and discussions. We also thank the Oberwolfach Mathematical Institute, where part of this work was done, for the hospitality. The article was finalized during the visit of A. N. at Princeton University. The research of E. G. was partially supported by the grants RFBR10-01-678, RFBR-13-01-00755, NSh-8462.2010.1, NSF grant DMS-1403560 and the Simons foundation. A. N. is partially supported by OTKA Grants 81203, 100796 and 112735.

\section{HeEgAard Floer LiNK HOMOLOGY}

2.1. Review of Heegaard Floer link homology. In this subsection we recall certain basic algebraic structures of Heegaard Floer link homology. For more see [15, 27, 28, 29, 30, 33]. 
To every 3-manifold $M$ with fixed Heegaard splitting one can associate a Heegaard Floer complex $C F^{-}(M)$ of free $\mathbb{Z}[U]$-modules. The operator $U$ has homological degree $(-2)$, and the differential $d$ has degree $(-1)$. This complex is not unique, but different choices (e.g. of a splitting) lead to quasi-isomorphic complexes. Therefore the homology of $C F^{-}(M)$ is an invariant of $M$ called Heegaard Floer homology and denoted by $H_{F}^{-}(M)$. For example, $H F^{-}\left(S^{3}\right)=\mathbb{Z}[U]$.

To a link $L=L_{1} \cup \cdots \cup L_{r} \subset S^{3}$ one can associate a $\mathbb{Z}^{r}$-filtered complex of $\mathbb{Z}\left[U_{1}, \ldots, U_{r}\right]$ modules, denoted by $C F L^{-}(L)$. If one ignores the filtration, then the complex is quasiisomorphic to the Heegaard Floer complex $C F^{-}\left(S^{3}\right)$, where all the operators $U_{i}$ are homotopic to each other, cf. [29]. One can also consider this complex as a $\mathbb{Z}[U]$-module, where $U=U_{1}$.

However, the filtration (called Alexander filtration) captures nontrivial information about the link. For $v \in \mathbb{Z}^{r}$, we will denote the Alexander filtration by $\left\{A^{-}(v)\right\}_{v}$. Each $A^{-}(v)=$ $\left(\oplus_{\nu} A^{-, \nu}(v), d\right)$ is a subcomplex of $C F L^{-}(L)$ (in [15] they are denoted by $\mathfrak{A}^{-}(v)$ ) 1 . The upper index $\nu$ denotes the homological (or Maslov) grading. They satisfy

$$
\begin{aligned}
& A^{-}(v) \supset A^{-}(u) \text { for } u \succ v, \text { and } \\
& A^{-}(v) \cap A^{-}(u)=A^{-}(\max \{v, u\}) .
\end{aligned}
$$

The subcomplexes $A^{-}(v)$ are $\mathbb{Z}\left[U_{1}, \ldots, U_{r}\right]$-submodules, the operators $U_{i}$ have homological degree $(-2)$ and are homotopic to each other. Moreover, $U_{i}\left(A^{-}(v)\right) \subset A^{-}\left(v+e_{i}\right)$.

The Heegaard Floer link homology is defined as the homology of the associated graded pieces of $A^{-}(v)$ :

$$
\operatorname{HFL}^{-}(L, v):=H_{*}\left(\left(\operatorname{gr} A^{-}\right)(v)\right), \text { where }\left(\operatorname{gr} A^{-}\right)(v):=A^{-}(v) / \sum_{u \succ v} A^{-}(u) .
$$

For example, for $r=1$ one has $\mathrm{HFL}^{-}(L, v)=H_{*}\left(A^{-}(v) / A^{-}(v+1)\right)$.

Remark 2.1.2. At present, Heegaard Floer link homology is defined only for $\mathbb{F}_{2}$ coefficients, hence, strictly speaking, all results of this section and the last section are valid only over $\mathbb{F}_{2}$. Nevertheless, we believe that all the statements are true over $\mathbb{Z}$ as well, but the cautious reader might take everywhere $\mathbb{F}_{2}$ instead of $\mathbb{Z}$.

By [29, Proposition 9.2], the Euler characteristic of the Heegaard Floer link homology coincides with the Reidemeister torsion, and it satisfies

$$
\sum_{v \in \mathbb{Z}^{r}} \chi\left(H F L^{-}(L, v)\right) \cdot t^{v}= \begin{cases}\Delta(t) & \text { if } r>1, \\ \frac{\Delta(t)}{1-t} & \text { if } r=1,\end{cases}
$$

where $\Delta$ is the multivariable Alexander polynomial of $L$.

2.2. $L$-space links. In [27] Ozsváth and Szabó introduced the notion of an $L$-space: a rational homology 3-sphere $M$ is an $L$-space if for any $\operatorname{spin}^{c}$-structure $s$ one has $\operatorname{rank} \widehat{H F}(M, s)=1$ (or, equivalently, $\mathrm{HF}^{-}(M, s)$ is a free $\mathbb{Z}[U]$-module of rank 1 ).

Definition 2.2.1. A link $L \subset S^{3}$ is called an $L$-space link if a sufficiently large surgery on all of its components is an $L$-space.

The following 'Large Surgery Theorem' shows the importance of the $L$-space property.

\footnotetext{
${ }^{1}$ For a more transparent match with the algebraic picture, we reverse the sign of $v$, thus reversing the direction of the filtration as well.
} 
Theorem 2.2.2. ([15, Theorem 10.1], see also [31, Lemma 4.2]) If $d_{1}, \ldots, d_{r}$ are sufficiently large integers, then the homology of $A^{-}(v)$ (considered as $\mathbb{Z}[U]$-module) is isomorphic to the Heegaard Floer homology $\mathrm{HF}^{-}$of the 3-manifold $S_{d_{1}, \ldots, d_{r}}^{3}(L)$ obtained from $S^{3}$ by $d_{i_{\text {-surgery }}}$ along the components of the link $L_{i}$ (for a certain $\operatorname{spin}^{c}-$ structure depending on $v$ ).

In particular, if $L \subset S^{3}$ is an L-space link, then for any $v \in \mathbb{Z}^{r}$ the homology of $A^{-}(v)$ is a free $\mathbb{Z}[U]$-module of rank 1 .

Let $\bar{g}(v)$ denote the homological degree of the unique generator in $H_{*}\left(A^{-}(v)\right)$.

Lemma 2.2.3. For all $i$ and $v \in \mathbb{Z}^{r}$ either $\bar{g}\left(v+e_{i}\right)=\bar{g}(v)$ or $\bar{g}\left(v+e_{i}\right)=\bar{g}(v)-2$. Furthermore, the inclusion map $A^{-}\left(v+e_{i}\right) \hookrightarrow A^{-}(v)$ induces an injection on homology.

Proof. One has the following inclusions:

$$
A^{-}(v) \supset A^{-}\left(v+e_{i}\right) \supset U_{i} A^{-}(v) \supset U_{i} A^{-}\left(v+e_{i}\right) .
$$

By Theorem 2.2.2, $H_{*}\left(A^{-}(v) / U_{i} A^{-}(v)\right)$ and $H_{*}\left(A^{-}\left(v+e_{i}\right) / U_{i} A^{-}\left(v+e_{i}\right)\right)$ are free $\mathbb{Z}$-modules of rank 1 with generators of homological degrees $\bar{g}(v)$ and $\bar{g}\left(v+e_{i}\right)$. Similarly to [27, Lemma 3.2] (see also [10]), from (2.2.4) one obtains the following alternative:

$$
\left\{\begin{array}{l}
\bar{g}\left(v+e_{i}\right)=\bar{g}(v) \text { and } \operatorname{dim} H_{*}\left(A^{-}(v) / A^{-}\left(v+e_{i}\right)\right)=0, \text { or } \\
\bar{g}\left(v+e_{i}\right)=\bar{g}(v)-2 \text { and } \operatorname{dim} H_{*}\left(A^{-}(v) / A^{-}\left(v+e_{i}\right)\right)=1 .
\end{array}\right.
$$

The long exact sequence in the homology implies the injectivity of the inclusion.

Motivated by Theorem 4.1.7 (valid for algebraic links) we introduce the following definition.

Definition 2.2.6. We define the $H F L$-weight function of an $L$-space link by

$$
g(v):=-\frac{1}{2} \bar{g}(v)
$$

Note that by Lemma 2.2.3 the values of $\bar{g}(v)$ have the same parity for all $v$, hence $g(v) \in \mathbb{Z}$ or $g(v) \in \frac{1}{2}+\mathbb{Z}$ for all $v$, hence $g\left(v+e_{i}\right)-g(v) \in\{0,1\}$.

Corollary 2.2.7. For all $u \succeq v$ the inclusion $i_{u v}: A^{-}(u) \hookrightarrow A^{-}(v)$ induces an injective map on homology. If $z(u)$ and $z(v)$ are generators in $H_{*}\left(A^{-}(u)\right)$ and in $H_{*}\left(A^{-}(v)\right)$ respectively, then

$$
i_{u v, *}(z(u))=U^{g(u)-g(v)} z(v) .
$$

Definition 2.2.8. Consider the "iterated cone" complex

$$
\mathcal{K}(v):=\bigoplus_{K \subset\{1, \ldots, r\}} A^{-}\left(v+e_{K}\right), \quad D=d+\sum_{i=1}^{r} \epsilon_{i, K} \partial_{i},
$$

where $d$ is a differential on $A^{-}, \partial_{i}: A^{-}\left(v+e_{K}\right) \rightarrow A^{-}\left(v+e_{K}-e_{i}\right)$ is the inclusion map $(i \in K)$, and the signs $\epsilon_{i, K}= \pm 1$ are chosen such that $D^{2}=0$.

It is useful to present $\mathcal{K}(v)$ as an $r$-dimensional cube with the complexes $\left\{A^{-}\left(v+e_{K}\right)\right\}_{K}$ at vertices. The differential $d$ acts in vertices, while $\partial:=\sum_{i=1}^{r} \epsilon_{i, K} \partial_{i}$ acts along the edges. The homological grading of a generator $x \in A^{-, \nu}\left(v+e_{K}\right)$, considered as a generator in $\mathcal{K}(v)$, equals $|K|+\nu$. The differential $d$ decreases $\nu$ by 1 and preserves $|K|$, the differential $\partial$ decreases $|K|$ by 1 and preserves $\nu$, so both decrease the total grading by 1 .

Lemma 2.2.9. The complexes $\left(\mathrm{gr} A^{-}\right)(v)$ and $\mathcal{K}(v)$ are quasi-isomorphic. 
Proof. We prove this by induction on $r$. For $r=1$ it is clear that $\mathcal{K}(v)$ is just the cone of the inclusion map $A^{-}\left(v+e_{1}\right) \rightarrow A^{-}(v)$, so it is quasi-isomorphic to $\left(\operatorname{gr} A^{-}\right)(v)=A^{-}(v) / A^{-}(v+$ $\left.e_{1}\right)$.

For $r>1$, we can write (using (2.1.1)

$$
\left.\left.\left(\operatorname{gr} A^{-}\right)(v)=\left(A^{-}(v) / \sum_{i=1}^{r-1} A^{-}\left(v+e_{i}\right)\right)\right) /\left(A^{-}\left(v+e_{r}\right) / \sum_{i=1}^{r-1} A^{-}\left(v+e_{i}+e_{r}\right)\right)\right) .
$$

Each of these quotients can be realized as an iterated cone, and $\left(\operatorname{gr} A^{-}\right)(v)$ can be realized as a cone of the natural map between them.

The following theorem and its proof is similar to the main result of [31], although it appears in a different setup. The algebraic construction of the 'iterated cone' complex $\mathcal{K}$ can be compared with the construction appearing in [31, Theorem 4.3].

For the definition of the lattice complex and cohomology see subsection 4.1 and section 4

Theorem 2.2.10. Let $L$ be an L-space link with $r$ components. Let us fix a point $v \in \mathbb{Z}^{r}$. There exists a spectral sequence with the following properties:

a) Its $E^{2}$ page is isomorphic (as graded $\mathbb{Z}$ module) to $H_{*}\left(\operatorname{gr}_{v} \mathcal{L}_{g}^{-}\right)$, where $\mathcal{L}_{g}^{-}$denotes the lattice complex associated with the HFL-weight function $g(v)$.

b) Its $E^{\infty}$ page is isomorphic (as graded $\mathbb{Z}$ module) to $\mathrm{HFL}^{-}(L, v)$, the Heegaard Floer link homology of $L$ with Alexander grading $v$.

c) The spectral sequence collapses at $E^{r}$ page (or earlier).

d) If $L$ has three or less components, then the spectral sequence collapses at $E^{2}$.

Proof. One has two (anti)commuting differentials $d$ and $\partial$ on the complex $\mathcal{K}(v)$, hence there exists a spectral sequence which starts with the cohomology of $d$ and converges to the cohomology of $D=d+\partial$. By Lemma 2.2.9, its (multigraded) $E^{\infty}$ page is isomorphic to

$$
E^{\infty}(v)=H_{*}(\mathcal{K}(v), D)=H_{*}\left(\left(\operatorname{gr} A^{-}\right)(v)\right)=H F L^{-}(L, v) .
$$

On the other hand, by Theorem 2.2.2, the $E^{1}$ page of this spectral sequence is isomorphic to

$$
E^{1}(v)=H_{*}(\mathcal{K}(v), d)=\bigoplus_{K} H_{*}\left(A^{-}\left(v+e_{K}\right)\right)=\bigoplus_{K} \mathbb{Z}[U] \cdot z\left(v+e_{K}\right),
$$

where, as above, we denote the generator in the homology of $A^{-}(u)$ by $z(u)$. One can naturally identify this $E^{1}$ page with the lattice complex $\left(\operatorname{gr}_{v} \mathcal{L}_{g}^{-}, \operatorname{gr} \partial_{U}\right)$, via the identification of $z\left(v+e_{K}\right)$ by $\square(v, K)$. Note that the $\nu$-grading of $z\left(v+e_{A}\right)$ (in $\mathcal{K}(v)$ ) equals $\bar{g}\left(v+e_{K}\right)=-2 g\left(v+e_{K}\right)$, hence the homological grading of $U^{m} z\left(v+e_{K}\right)$ equals $\nu\left(U^{m} z\left(v+e_{K}\right)\right)+|K|=-2 m-2 g(v+$ $\left.e_{K}\right)+|K|$, in agreement with the definition of the homological degree in the lattice complex in 4.1, see also (4.1.4). The next differential is induced by $\partial$, and by Corollary 2.2.7 it agrees with the lattice differential for the weight function $g(v)$. Indeed,

$$
\partial\left(z\left(v+e_{K}\right)\right)=\sum_{i \in K} \pm \partial_{i}\left(z\left(v+e_{K}\right)\right)=\sum_{i \in K} \pm U^{g\left(v+e_{K}\right)-g\left(v+e_{K}-e_{i}\right)} z\left(v+e_{K}-e_{i}\right) .
$$

The differential $d_{k}$ in the spectral sequence decreases $|K|$ by $k$ and increases the $\nu$-grading (homological grading in vertices of the cube) by $k-1$ (assuming that $d=d_{0}$ and $\partial=d_{1}$ ). In particular, for $k>r$ the differential $d_{k}$ vanishes automatically. Moreover, the class of the unique $r$-dimensional cube is not in the kernel of the lattice differential, so $d_{r}$ vanishes too.

Since the $\nu$-gradings of all classes on $E^{1}$ page has the same parity, $d_{k}$ can be nontrivial only if $k$ is odd. In particular, for $r \leq 3$ we have $d_{2}=d_{3}=0$, so $E^{2}=E^{\infty}$. 
The next theorem expresses the function $g(v)$ in terms of the Euler characteristic of the $H F L^{-}$homology (or, equivalently, in terms of multivariable Alexander polynomial).

Theorem 2.2.11. Let $L_{K}$ denote the sublink associated with $K \subset K_{0}$. Then for every $v \in \mathbb{Z}^{r}$

$$
g(v)=\sum_{K \subset K_{0}}(-1)^{|K|-1} \sum_{0 \preceq u \preceq v_{K}-e_{K}} \chi\left(H F L^{-}\left(L_{K}, u\right)\right) .
$$

Proof. Since the Heegaard Floer complex is finitely generated as $\mathbb{Z}[U]$-module, there exists $N=\left(N_{1}, \ldots, N_{r}\right)$ large enough such that $A^{-}(v) \subset A^{-}(-N)$ for any $v$. Hence

$$
g(v)=g(\max \{v,-N\}) .
$$

For a subset $K=\left\{i_{1}, \ldots, i_{|K|}\right\} \subset K_{0}$ consider a sublink $L_{K}:=\cup_{i \in K} L_{i} . \quad L_{K}$ is also $L-$ space link (cf. [14, Lemma 1.6]), so it defines a $H F L$-weight function $g_{K}$ on the sublattice of $\mathbb{Z}^{r}$ supported on $K$. By [29, Proposition 7.1], the restriction of the filtration $A^{-}(v)$ to this sublattice coincides with the filtration on the Heegaard Floer complex for the sublink $L_{K}$. Given $v_{i_{1}}, \ldots, v_{i_{|K|}}$, define

$$
u\left(v_{i_{1}}, \ldots, v_{i_{|K|}}\right):= \begin{cases}v_{j}, & j \in K, \\ -N_{j}, & j \notin K,\end{cases}
$$

then $A^{-}\left(u\left(v_{i_{1}}, \ldots, v_{i_{|K|}}\right)\right) \simeq A_{L_{K}}^{-}\left(v_{i_{1}}, \ldots, v_{i_{|K|}}\right)$ and

$$
g\left(u\left(v_{i_{1}}, \ldots, v_{i_{|K|}}\right)\right)=g_{K}\left(v_{i_{1}}, \ldots, v_{i_{|K|}}\right) .
$$

At Euler characteristic level we obtain

$$
\chi\left(H F L^{-}\left(L_{K}, v\right)\right)=\chi\left(A^{-}(v) / \sum_{i \in K} A^{-}\left(v+e_{i}\right)\right)=\sum_{M \subset K}(-1)^{|M|-1} g\left(v+e_{M}\right) .
$$

This is a linear system of equations for $g(v)$, and by Theorem 3.4 .3 (where 0 should be replaced by $-N)$ the function $g(v)$ is defined uniquely by the equations (2.2.12) and (2.2.13) up to an overall shift.

\section{THE HILBERT FUNCTION AND ITS RELATION WITH OTHER INVARIANTS}

In this section we discuss the connections between the multi-variable Alexander polynomial, three series (Poincaré, Hilbert and motivic Poincaré), and the semigroup associated with an isolated plane curve singularity. All the statements, except those which involve the Alexander polynomial, are valid for arbitrary (non necessarily plane) curve singularity germs. The Alexander polynomial, by its very essence, is an invariant of the embedded topological type (hence of the embedded link); in the algebraic case it connects the theory of links of $S^{3}$ with the above algebraic invariants.

3.1. The Hilbert series of the multi-index filtration. We fix a local reduced plane curve singularity with $r$ irreducible components $C_{i}$ and normalizations $\gamma_{i}:(\mathbb{C}, 0) \rightarrow\left(C_{i}, 0\right)$. Set the valuations $\mathfrak{v}_{i}(f)=\operatorname{ord}_{t}\left(f\left(\gamma_{i}(t)\right)\right)$ on $\mathcal{O}=\mathcal{O}_{\mathbb{C}^{2}, 0}$, and a $\mathbb{Z}^{r}$-indexed filtration

$$
J(v)=\{f \in \mathcal{O} \mid \mathfrak{v}(f) \succeq v\} .
$$

Note that the ideals $J(v)$ are defined for negative values of $v$ as well. The filtration is decreasing: if $u \preceq v$ then $J(u) \supset J(v)$.

Definition 3.1.1. The Hilbert series of the multi-index filtration $J$ is

$$
H\left(t_{1}, \ldots, t_{r}\right)=\sum_{v} h(v) \cdot t_{1}^{v_{1}} \cdots t_{r}^{v_{r}} \in \mathbb{Z}\left[\left[t_{1}, t_{1}^{-1}, \ldots, t_{r}, t_{r}^{-1}\right]\right],
$$


where $h(v)=\operatorname{dim}_{\mathbb{C}} \mathcal{O} / J(v)$. Note that

$$
h(v)=h(\max \{v, 0\}) .
$$

Hence $H$ is determined completely by $\left.H(t)\right|_{0 \preceq v}:=\sum_{0 \preceq v} h(v) t^{v}$.

3.2. The Poincaré series. If $r=1$, then the Poincaré series of the graded ring $\oplus_{v} J(v) / J(v+$ $\left.e_{1}\right)$ is $P(t)=-H(t)\left(1-t^{-1}\right)$. For general $r$, one defines the Poincaré series similarly

$$
P\left(t_{1}, \ldots, t_{r}\right)=-H\left(t_{1}, \ldots, t_{r}\right) \cdot \prod_{i}\left(1-t_{i}^{-1}\right) .
$$

This means that the coefficient $\pi_{v}$ of $P=\sum_{v} \pi_{v} \cdot t_{1}^{v_{1}} \ldots t_{r}^{v_{r}}$ satisfies

$$
\pi_{v}=\sum_{K \subset K_{0}}(-1)^{|K|-1} h\left(v+e_{K}\right) .
$$

The space $\mathbb{Z}\left[\left[t_{1}, t_{1}^{-1}, \ldots, t_{r}, t_{r}^{-1}\right]\right]$ is a module over the ring of Laurent power series, hence the multiplication in (3.2.1) is a well-defined. One can check (using e.g. (3.1.3) ) that the right hand side of (3.2.1) is a power series involving only nonnegative powers of $t_{i}$.

3.3. Poincaré series and the Alexander polynomial. The topological aspect and importance of the Poincaré series is shown by the following theorem.

Theorem 3.3.1 ([4, 5]). Let $\Delta\left(t_{1}, \ldots, t_{r}\right)$ be the multi-variable Alexander polynomial of the link of $C$. If $r=1$ then $P(t)(1-t)=\Delta(t)$, while $P\left(t_{1}, \ldots, t_{r}\right)=\Delta\left(t_{1}, \ldots, t_{r}\right)$ if $r>1$.

The Alexander polynomial is symmetric in the following sense. For any $i \in K_{0}$ let $\mu_{i}$ and $\delta_{i}$ (respectively $\mu(C)$ and $\delta(C)$ ) be the Milnor number and the delta invariant of $C_{i}$ (respectively of $C)$, see [1, 16]. Let $\left(C_{j}, C_{i}\right)$ be the intersection multiplicities at $0(j \neq i)$. Then, cf. [16], $\mu_{i}=2 \delta_{i}$, and $\mu(C)+r-1=2 \delta(C)$. Define $l=\left(l_{1}, \ldots, l_{r}\right)$ by

$$
l_{i}=\mu_{i}+\sum_{j \neq i}\left(C_{j}, C_{i}\right) \quad(1 \leq i \leq r)
$$

Then $\Delta\left(t^{-1}\right)=t^{-\mu(C)} \Delta(t)$ for $r=1$, and (e.g. by [9])

$$
\Delta\left(t_{1}^{-1}, \ldots, t_{r}^{-1}\right)=\left(\prod t_{i}^{1-l_{i}}\right) \cdot \Delta\left(t_{1}, \ldots, t_{r}\right) \text { for } r>1 .
$$

By [7, 18], the Hilbert function also satisfies similar symmetry properties

$$
h(l-v)-h(v)=\delta(C)-|v|,
$$

where $|v|=\sum_{i=1}^{r} v_{i}$. In particular, for $v \succeq l$ one has

$$
h(v)=|v|-\delta(C) .
$$

3.4. The equivalence of the Poincaré series and the Hilbert series. For any subset $K=$ $\left\{i_{1}, \ldots, i_{|K|}\right\} \subset K_{0}, K \neq \emptyset$, consider the curve $C_{K}=\cup_{i \in K} C_{i}$. As above, this germ defines the Hilbert series $H_{C_{K}}$ of $C_{K}$ in variables $\left\{t_{i}\right\}_{i \in K}$ :

$$
H_{C_{K}}\left(t_{i_{1}}, \ldots, t_{i_{|K|}}\right)=\sum_{v} h^{K}(v) \cdot t_{i_{1}}^{v_{i_{1}}} \ldots t_{i_{|K|}}^{v_{i_{|K|}}} .
$$

By the very definition, $H_{C_{K}}\left(t_{i_{1}}, \ldots, t_{i_{|K|}}\right)=\left.H_{C}\left(t_{1}, \ldots, t_{r}\right)\right|_{t_{i}=0 i \notin K}$; or

$$
\text { if } v_{i}=0 \text { for all } i \notin K \text {, then } h^{K}(v)=h(v) \text {. }
$$


Analogously, we also consider the Poincaré series of $C_{K}$ :

$$
P_{C_{K}}\left(t_{i_{1}}, \ldots, t_{i_{|K|}}\right)=\sum_{v} \pi_{v}^{K} \cdot t_{i_{1}}^{v_{i_{1}}} \ldots t_{i_{|K|}}^{v_{i_{|K|}}} .
$$

By definition, for $K=\emptyset$ we take $\pi_{v}^{\emptyset}=0$.

By [36] the multi-variable Alexander polynomial (and hence by Theorem 3.3.1 the Poincare series $P(t)$ ) determines the embedded topological type of $C$, in particular all the series $\left\{P_{C_{K}}\right\}_{K \subset K_{0}}$. Nevertheless, the reduction procedure from $P$ to $P_{C_{K}}$ is more complicated than the analogs of (3.4.1) valid for the Hilbert series. Indeed, these formulae are of type (see [35]):

$$
P_{C_{K_{0} \backslash\{1\}}}\left(t_{2}, \ldots, t_{r}\right)=\left.P\left(t_{1}, \ldots, t_{r}\right)\right|_{t_{1}=1} \cdot \frac{1}{\left(1-t_{2}^{\left(C_{1}, C_{2}\right)}\right) \cdots\left(1-t_{r}^{\left(C_{1}, C_{r}\right)}\right)} .
$$

The next theorem inverts (3.2.2): we recover $H$ from $P$. The fact that $H$ can be recovered from $P$ was already proved in [17, Corollary 4.3]. However, we wish to present a more general statement which also clarifies under what condition the inversion works, and which is applied for certain coefficients provided by the Heegaard Floer link homology as well, cf. Theorem 2.2.10 and identity (2.2.13).

Theorem 3.4.3. Consider $G\left(t_{1}, \ldots, t_{r}\right)=\sum_{v} t_{1}^{v_{1}} \ldots t_{r}^{v_{r}} \cdot g(v) \in \mathbb{Z}\left[\left[t_{1}, t_{1}^{-1}, \ldots, t_{r}, t_{r}^{-1}\right]\right]$ with the following properties:

(a) $g(v)=g(\max \{v, 0\})$;

(b) $g(0)=0$.

(c) Fix $K \subset K_{0}$. We extend any $v=\left(v_{i_{1}}, \ldots, v_{i_{|K|}}\right)$ to a vector with entries indexed by $K_{0}$ such that the entries indexed by $K_{0} \backslash K$ are zero. (In this way $g(v)$ make sense.) Then, we also require that the coefficients of $g$ satisfy (for any $K$ ) the following identities:

$$
\pi_{v}^{K}=\sum_{M \subset K}(-1)^{|M|-1} g\left(v+e_{M}\right) \quad \text { for any } v=\left(v_{i_{1}}, \ldots, v_{i_{|K|}}\right) .
$$

Then $G$ is uniquely determined by $\left\{P_{C_{K}}\right\}_{K}$ (hence by $P$ too), and it satisfies

$$
\left.G\left(t_{1}, \ldots, t_{r}\right)\right|_{0 \preceq v}=\frac{1}{\prod_{i=1}^{r}\left(1-t_{i}\right)} \sum_{K \subset K_{0}}(-1)^{|K|-1}\left(\prod_{i \in K} t_{i}\right) \cdot P_{C_{K}}\left(t_{i_{1}}, \ldots, t_{i_{|K|}}\right) .
$$

Proof. The identity (3.4.4) is equivalent to the following identity of the coefficients:

$$
g(v)=\sum_{K \subset K_{0}}(-1)^{|K|-1} \sum_{0 \preceq u \preceq v_{K}-e_{K}} \pi_{u}^{K} .
$$

We will prove the identity (3.4.5) by a two-step induction: the first induction is by the number of components $r$, and the second one (for fixed $r$ ) is over the norm $|v|=\sum v_{i}$.

If $r=1$, then (d) implies $\pi_{v}=g(v+1)-g(v)$. Hence $\sum_{0 \leq u \leq v-1} \pi_{u}=g(v)$ since $g(0)=0$.

Let us prove (3.4.5) for the case when at least one of coordinates $v_{i}$ vanish. We can assume that $v_{r}=0$. By (3.4.1) and the induction assumption we get

$$
g(v)=g\left(v_{1}, \ldots, v_{r-1}, 0\right)=\sum_{K \subset\{1, \ldots, r-1\}}(-1)^{|K|-1} \sum_{0 \preceq u \preceq v_{K}-e_{K}} \pi_{u}^{K} .
$$

On the other hand, in (3.4.5) for all $K \subset K_{0}$ with $r \in K$ we get the vacuous restriction $0 \leq u_{r} \leq-1$, hence we get a nontrivial contribution only from terms with $K \subset\{1, \ldots, r-1\}$.

Suppose now that $v$ has no vanishing coordinates and that we already proved (3.4.5) for $v-e_{K}$ for all non-empty subsets $K \subset K_{0}$. We can rewrite (d) as a linear equation on $\{g(v-$ 
$\left.\left.e_{K}\right)\right\}_{K}$ :

$$
\pi_{v-e}=\sum_{K \subset K_{0}}(-1)^{r-|K|-1} g\left(v-e_{K}\right)
$$

By the induction assumption for $K \neq \emptyset$ we have

$$
g\left(v-e_{K}\right)=\sum_{M \subset K_{0}}(-1)^{|M|-1} \sum_{0 \preceq u \preceq\left(v_{M}-e_{K \cap M}-e_{M}\right)} \pi_{u}^{M},
$$

and we should establish the same identity for $K=\emptyset$. Therefore we need to prove that

$$
\pi_{v-e}=\sum_{K \subset K_{0}} \sum_{M \subset K_{0}}(-1)^{r-|K|+|M|} \sum_{0 \preceq u \preceq\left(v_{M}-e_{K \cap M}-e_{M}\right)} \pi_{u}^{M} .
$$

Let us fix $M$ and $u \preceq v-e$ and sum the expression $(-1)^{|K|}$ over all sets $K \subset K_{0}$ such that $u_{i} \leq v_{i}-2$ for $i \in K \cap M$. This sum vanishes unless $M=K_{0}$ and $u_{i}=v_{i}-1$ for all $i$, when it is 1 . This proves (3.4.6).

Corollary 3.4.7. (a) The Hilbert series satisfies the assumptions of the above inversion theorem, hence $G=H$.

(b) The restricted Hilbert function $\left.H(t)\right|_{0 \preceq v}$ of a multi-component curve is a rational function with denominator $\prod_{i=1}^{r}\left(1-t_{i}\right)^{2}$.

Proof. For (a) use identities (3.4.1) and (3.2.2) applied for $C_{K}$, while for (b) Theorems 3.4.3 and 3.3 .1 .

Remark 3.4.8. Let us reprove the identity (3.3.3) using (3.4.5). We analyze the different contributions. For $K=\{i\}$ we have $\sum_{0 \leq u_{i} \leq v_{i}-1} \pi_{u}^{K}=v_{i}-\delta\left(C_{i}\right)$. For $K=\{i, j\}$ (since $P_{C_{K}}$ is a polynomial) we have $\sum \pi_{u}^{\{i, j\}}=P_{C_{K}}(1,1)$. This equals $\left(C_{i}, C_{j}\right)$ by (3.4.2). By similar argument, for $|K|>2$ the contribution is zero. Hence $h(v)=\sum_{i}\left(v_{i}-\delta\left(C_{i}\right)\right)-\sum_{i \neq j}\left(C_{i}, C_{j}\right)=$ $|v|-\delta(C)$.

3.5. The semigroup of $C$. Important information about the algebraic curve $C$ is coded in its semigroup. It is defined as $\mathcal{S}:=\left\{v \in \mathbb{Z}^{r} \mid\right.$ there exists $f \in \mathcal{O}$ with $\left.\mathfrak{v}(f)=v\right\}$.

Lemma 3.5.1. The semigroup can be equivalently defined by the following condition:

$$
\mathcal{S}=\left\{v \in \mathbb{Z}_{\geq 0}^{r} \mid h\left(v+e_{i}\right)>h(v) \text { for every } i=1, \ldots, r\right\} .
$$

Next, fix any $0 \preceq v$ and $e_{i}$. Then $h\left(v+e_{i}\right)=h(v)+1$ if there is an element $u \in \mathcal{S}$ such that $u_{i}=v_{i}$ and $u_{j} \geq v_{j}$ for $j \neq i$. Otherwise $h\left(v+e_{i}\right)=h(v)$.

In particular, $H$ and $\mathcal{S}$ determine each other.

Proof. If $h\left(v+e_{i}\right)>h(v)$ for all $i$, then there exist functions $f_{i}$ such that $\mathfrak{v}_{i}\left(f_{i}\right)=v_{i}$ and $\mathfrak{v}_{j}\left(f_{i}\right) \geq v_{j}$ for $j \neq i$. Therefore $\mathfrak{v}\left(\sum_{i=1}^{r} \lambda_{i} f_{i}\right)=v$ for generic coefficients $\lambda_{i}$. For the second part note that $h\left(v+e_{i}\right)-h(v)=\operatorname{dim} J(v) / J\left(v+e_{i}\right)$. This quotient space is trivial if there is no function $f$ such that $\mathfrak{v}_{i}(f)=v_{i}$ and $\mathfrak{v}_{j}(f) \geq v_{j}$ for $j \neq i$. Otherwise it is one-dimensional. Indeed, if $\mathfrak{v}_{i}\left(f_{1}\right)=\mathfrak{v}_{i}\left(f_{2}\right)=v_{i}$ then there exists $\lambda \neq 0$ such that $\mathfrak{v}_{i}\left(f_{1}-\lambda f_{2}\right)>v_{i}$. If, moreover, $\mathfrak{v}_{j}\left(f_{1}\right), \mathfrak{v}_{j}\left(f_{2}\right) \geq v_{j}$ for all $j \neq i$, then $\mathfrak{v}_{j}\left(f_{1}-\lambda f_{2}\right) \geq v_{j}$ too. Therefore $f_{1}-\lambda f_{2} \in J\left(v+e_{i}\right)$.

Next we establish the 'matroid properties' of the function $h$.

Lemma 3.5.2. (a) Assume that $h(v)=h\left(v+e_{i}\right)$ for some fixed $i \in K_{0}$. Then $h\left(v+e_{K}\right)=$ $h\left(v+e_{K}+e_{i}\right)$ for any $K$ with $K \not \supset i$.

(b) Suppose that $K_{1}, K_{2} \subset K_{0}$ and $v \in \mathbb{Z}^{r}$. Then

$$
h\left(v+e_{K_{1}}\right)+h\left(v+e_{K_{2}}\right) \geq h\left(v+e_{K_{1} \cap K_{2}}\right)+h\left(v+e_{K_{1} \cup K_{2}}\right) .
$$




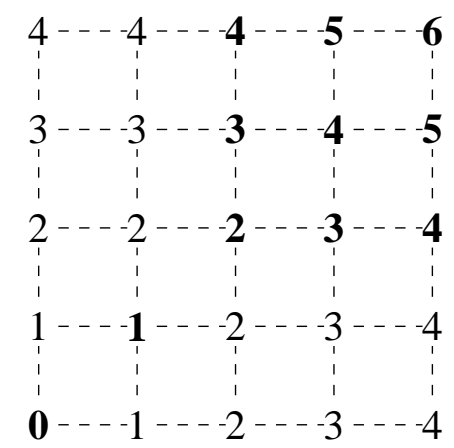

FIGURE 1. Values of the Hilbert function for $A_{3}$

(c) For any base vector $e_{i}$ and $n \geq l_{i}$ one has $h\left(v+(n+1) e_{i}\right)-h\left(v+n e_{i}\right)=1$.

Proof. (a) Use $J\left(v+e_{K}+e_{i}\right)=J\left(v+e_{K}\right) \cap J\left(v+e_{i}\right)$.

(b) Replacing $v$ by $v+e_{K_{1} \cap K_{2}}$, we can assume that $K_{1} \cap K_{2}=\emptyset$. Therefore, $J\left(v+e_{K_{1}}\right) \cap$ $J\left(v+e_{K_{2}}\right)=J\left(v+e_{K_{1} \cup K_{2}}\right)$. Hence $h\left(v+e_{K_{1}}\right)+h\left(v+e_{K_{2}}\right)-h(v)-h\left(v+e_{K_{1} \cup K_{2}}\right)=$ $\operatorname{dim} J(v) /\left(J\left(v+e_{K_{1}}\right)+J\left(v+e_{K_{2}}\right)\right) \geq 0$. For (c) use (3.3.3) and Lemma 3.5.1.

Remark 3.5.3. It turns out (using e.g. (3.3.3) and Lemma 3.5.1) that $l$ is the conductor of $\mathcal{S}$, in particular $v \in \mathcal{S}$ whenever $v \succeq l$.

Example 3.5.4. Consider the singularity $A_{2 n-1}$ defined by the equation $x^{2}-y^{2 n}=0$. Its Poincaré series equals $1+t_{1} t_{2}+\cdots+\left(t_{1} t_{2}\right)^{n-1}$, and the Poincaré series of both its components equals $1 /(1-t)$. The Hilbert series is given by the following equation:

$$
\left.H\left(t_{1}, t_{2}\right)\right|_{0 \preceq v}=\frac{1}{\left(1-t_{1}\right)\left(1-t_{2}\right)}\left(\frac{t_{1}}{1-t_{1}}+\frac{t_{2}}{1-t_{2}}-t_{1} t_{2}\left(1+\ldots+\left(t_{1} t_{2}\right)^{n-1}\right)\right) .
$$

Therefore, for non-negative integers $\left(v_{1}, v_{2}\right)$ one has

$$
h(v)= \begin{cases}\max \left(v_{1}, v_{2}\right), & \text { if } \min \left(v_{1}, v_{2}\right)<n, \\ v_{1}+v_{2}-n, & \text { otherwise. }\end{cases}
$$

Figure 1 illustrates this formula for the Hilbert function for $A_{3}$ singularity. The points corresponding to the semigroup $\mathcal{S}$ are marked in bold.

Example 3.5.5. Consider the singularity $D_{5}$ defined by the equation $y \cdot\left(x^{2}-y^{3}\right)=0$. Then

$$
P\left(t_{1}, t_{2}\right)=1+t_{1} t_{2}^{3}, \quad P_{1}\left(t_{1}\right)=\frac{1}{1-t_{1}}, \quad P_{2}\left(t_{2}\right)=\frac{1-t_{2}+t_{2}^{2}}{1-t_{2}} .
$$

One can check that $h\left(v_{1}, v_{2}\right)$ for non-negative $v_{1}$ and $v_{2}$ is given by the following formula:

$$
h\left(v_{1}, v_{2}\right)= \begin{cases}v_{1}, & \text { if } v_{2}<3, v_{1}>0 \\ v_{1}+1, & \text { if } v_{2}=3, v_{1}>0 \\ v_{2}-1, & \text { if } v_{1}<2, v_{2} \geq 2 \\ v_{1}+v_{2}-3, & \text { if } v_{1} \geq 2, v_{2} \geq 4 \\ 0,1,1, & \text { if } v_{1}=0 \text { and } v_{2}=0,1,2 .\end{cases}
$$

Figure 2 illustrates the Hilbert function and the semigroup of $D_{5}$. 


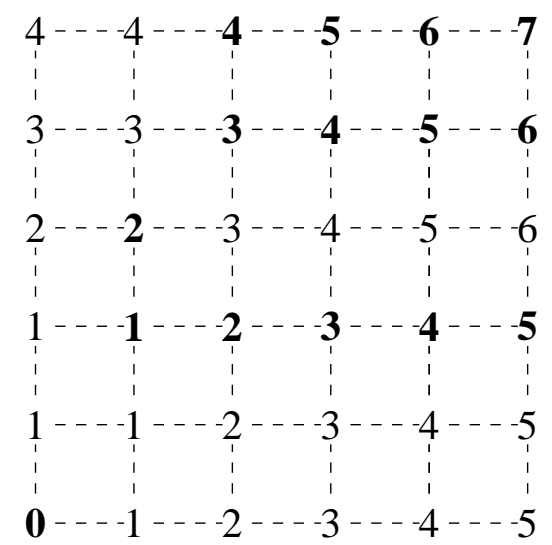

FIGURE 2. Values of the Hilbert function for $D_{5}$

3.6. The local hyperplane arrangements. For any fixed $v$ let us consider the set

$$
\mathcal{H}(v):=\{f \in \mathcal{O}: \mathfrak{v}(f)=v\}=J(v) \backslash \bigcup_{i} J\left(v+e_{i}\right) .
$$

Since $J\left(v+e_{i}\right)$ is either $J(v)$ or one of its hyperplanes (cf. 3.5.1), $\mathcal{H}(v)$ is either empty or it is a hyperplane arrangement in $J(v)$. This can be reduced to a finite dimensional central hyperplane arrangement

$$
\mathcal{H}^{\prime}(v):=\frac{J(v)}{J\left(v+e_{K_{0}}\right)} \backslash \bigcup_{i} \frac{J\left(v+e_{i}\right)}{J\left(v+e_{K_{0}}\right)},
$$

since $\mathcal{H}(v) \simeq J\left(v+e_{K_{0}}\right) \times \mathcal{H}^{\prime}(v)$. Note that both $\mathcal{H}^{\prime}(v)$ and $\mathcal{H}(v)$ admit a free $\mathbb{C}^{*}$-action (multiplication by nonzero scalar), hence one automatically has the two projective arrangements $\mathbb{P} \mathcal{H}^{\prime}(v)=\mathcal{H}^{\prime}(v) / \mathbb{C}^{*}$ and $\mathbb{P} \mathcal{H}(v)=\mathcal{H}(v) / \mathbb{C}^{*}$ as well. The following proposition can be deduced from (3.2.2) and inclusion-exclusion formula (see e.g. [4, 5] and Lemma 3.7.1 below).

Proposition 3.6.1. The Euler characteristic of $\mathbb{P} \mathcal{H}(v)$ (and of $\mathbb{P} \mathcal{H}^{\prime}(v)$ ) equals $\pi_{v}$, the coefficient of the Poincaré series $P(t)$ at $t^{v}$.

3.7. Motivic Poincaré series. The series $\mathcal{P}\left(t_{1}, \ldots, t_{r} ; q\right) \in \mathbb{Z}\left[\left[t_{1}, \ldots, t_{r}\right]\right][q]$ is defined in [6] as a refinement of $P(t)$ as follows. By definition, the coefficient of $t_{1}^{v_{1}} \ldots t_{r}^{v_{r}}$ is the (normalized) class of $\mathbb{P} \mathcal{H}^{\prime}(v)$ in the Grothendieck ring of algebraic varieties. It turns out that the class of a central hyperplane arrangement can always be expressed in terms of the class $\mathbb{L}$ of the affine line. Indeed, one has:

Lemma 3.7.1. $V$ be a vector space and let $\mathcal{H}=\left\{\mathcal{H}_{1}, \ldots, \mathcal{H}_{r}\right\}$ be a collection of linear hyperplanes in $V$. For a subset $K$ we define the rank function by $\rho(K)=\operatorname{codim} \cup_{i \in K} \mathcal{H}_{i}$. Then in the Grothendieck ring of varieties (by the inclusion-exclusion formula) one has

$$
\left[V \backslash \cup_{i=1}^{r} \mathcal{H}_{i}\right]=\sum_{K \subset K_{0}}(-1)^{|K|}\left[\cap_{\alpha \in K} \mathcal{H}_{\alpha}\right]=\sum_{K \subset K_{0}}(-1)^{|K|} \mathbb{L}^{\operatorname{dim} V-\rho(K)} .
$$

Since $\left[\mathbb{C}^{*}\right]=\mathbb{L}-1$, one also has $\left[\left(V \backslash \cup_{i=1}^{r} \mathcal{H}_{i}\right) / \mathbb{C}^{*}\right]=\left[V \backslash \cup_{i=1}^{r} \mathcal{H}_{i}\right] /(\mathbb{L}-1)$.

Corollary 3.7.2. The class of the (finite) local hyperplane arrangement $\mathcal{H}^{\prime}(v)$ equals

$$
\left[\mathcal{H}^{\prime}(v)\right]=(\mathbb{L}-1)\left[\mathbb{P} \mathcal{H}^{\prime}(v)\right]=\sum_{K \subset K_{0}}(-1)^{|K|} \mathbb{L}^{h\left(v+e_{K_{0}}\right)-h\left(v+e_{K}\right)} .
$$


Replacing $\mathbb{L}^{-1}$ by a new variable $q$, one can define (following [6]) the motivic Poincaré series $\mathcal{P}(t ; q)=\sum_{v} \pi_{v}(q) t^{v}$ by

$$
\begin{gathered}
\pi_{v}(q):=\left.\mathbb{L}^{1-h\left(v+e_{K_{0}}\right)}\left[\mathbb{P} \mathcal{H}^{\prime}(v)\right]\right|_{\mathbb{L}^{-1}=q}=\frac{1}{1-q} \sum_{K \subset K_{0}}(-1)^{|K|} q^{h\left(v+e_{K}\right)}= \\
\sum_{K \subset K_{0}}(-1)^{|K|} \cdot \frac{q^{h\left(v+e_{K}\right)}-q^{h(v)}}{1-q} .
\end{gathered}
$$

Note that $\lim _{q \rightarrow 1} \mathcal{P}(t ; q)=P(t)$. In [10, 18] several properties of $\mathcal{P}\left(t_{1}, \ldots, t_{r} ; q\right)$ are proved, e.g. it is a rational function with denominator $\prod_{i=1}^{r}\left(1-t_{i} q\right)$. We will need the following.

Lemma 3.7.3. The support of $\mathcal{P}(t ; q)$ is exactly $\mathcal{S}$. That is, $\pi_{v}(q) \neq 0$ if and only if $v \in \mathcal{S}$.

Proof. If $v \notin \mathcal{S}$, then there exists $i \in K_{0}$ such that $h\left(v+e_{i}\right)=h(v)$ (cf. 3.5.1), hence $\pi_{v}(q)=0$ by 3.5 .2 (a). If $v \in S$, then $h\left(v+e_{i}\right)=h(v)+1$ for all $i$ and $h\left(v+e_{K}\right) \geq h(v)+1$ for all subsets $K$, hence $\pi_{v}(q)=q^{h(v)}+$ higher order terms.

By Theorem 3.4.3, $\mathcal{P}(t ; q)$ and $P(t)$, in fact, determine each other.

3.8. Conclusion. By the above discussions, the following objects associated with a plane curve singularity carry the same amount of information: the multi-variable Alexander polynomial $\Delta(t)$, the semigroup $\mathcal{S}$, the Hilbert series $H(t)$, the Poincare series $P(t)$ and the motivic Poincare series $\mathcal{P}(t ; q)$. The role of the spaces $\mathcal{H}(v)$ will be crucial in the next parts: we will compute their homology using the Orlik-Solomon algebras of hyperplane arrangements. This will connect two other objects: the local lattice homology (associated with the weight function $h$ ) and the Heegaard Floer link homology of the link of $C$. This connection and the 'matroid properties' (3.5.2) of the weight function $h$ are responsible for the collapse of a spectral sequence connecting the Heegaard Floer link homology with the lattice homology.

The Poincaré polynomials of all these cohomologies will be identified with the coefficients of the motivic Poincare series.

Remark 3.8.1. In the above definition $\mathcal{O}_{\mathbb{C}^{2}, 0}$ can be replaced by $\mathcal{O}_{C}$. In this way, one can extend all the above definitions of $H(t), P(t), \mathcal{P}(t ; q), \mathcal{S}$ to the case of any (not necessarily plane) reduced curve singularity. The topological embedded-link invariant $\Delta(t)$ has no analogue in this general case. It is a nice challenge to find the analogue of the $H F L$-theory (via $(H(t)$ as in this note) applied for a (non-planar) curve singularity.

\section{LATTICE HOMOLOGY}

Lattice homology associated with the intersection lattice of a resolution of a normal surface singularity was introduced in [23], as a topological invariant of negative definite plumbed 3manifolds. For a possible generalization to algebraic knots, see the recent manuscript [32].

In this section we introduce another homology theory associated with curve singularities, where the lattice and the corresponding weight function have a different nature. In order to make a distinction between the two cases we will call the present theory lattice homology of curve singularities via their normalizations. In fact, the definitions below extend identically to any, not necessarily plane curve singularity, that is, even if $(C, 0)$ does not have any local embedded link in the 3-sphere. 


\subsection{The general theory: lattice complex, filtrations, lattice homology.}

In this subsection we present the general theory of lattice homology associated with an arbitrary weight function. This will be specialized for the function $h$ in subsection 4.2, and for the $H F L$-weight function $g$ given by Heegaard Floer link theory in section 6 (see also section 2 ).

We will use the cubes in $\mathbb{R}^{r}$ with vertices in the lattice points $\mathbb{Z}^{r}$. Every such cube $\mathbf{\square}(v, K)$, where $v \in \mathbb{Z}^{r}$ and $K \subset K_{0}$, is defined as

$$
\mathbf{\square}=\mathbf{\square}(v, K)=\left\{x \in \mathbb{R}^{r}: v \preceq x \preceq v+e_{K}\right\}, \operatorname{dim} \mathbf{\square}(v, K)=|K| .
$$

We consider $\mathbf{\square}$ with its natural orientation (as a subset of $\mathbb{R}^{r}$ ). In the classical cubical homology, the chain complex is a free $\mathbb{Z}$-module with generators $\square=\square(v, K)$ corresponding to the cubes $\mathbf{\square}(v, K)$, and the differential can be written as $\partial(\square)=\sum_{i} \varepsilon_{i} \square_{i}$, where $\mathbf{\square}_{i}$ are oriented codimension 1 faces of the cube $\mathbf{\square}$. 2

Definition 4.1.1. Let us choose a function $w: \mathbb{Z}^{r} \rightarrow \mathbb{Z}$, which will be called weight function. We define the weight of a cube by

$$
w(\square)=\max \left\{w(v): v \in \mathbf{\square} \cap \mathbb{Z}^{r}\right\} .
$$

If $w(v)$ is non-decreasing (that is, $w(u) \leq w(v)$ whenever $u \preceq v$ ), then, in fact, $w(\square(v, K))=$ $w\left(v+e_{K}\right)$.

Definition 4.1.2. The lattice complex $\mathcal{L}_{w}^{-}$associated with a weight function $w$ is a free $\mathbb{Z}[U]$ module generated by all cubes $\square=\square(v, K)$ with the following $\mathbb{Z}[U]$-linear differential:

$$
\partial_{U}(\square)=\sum_{i} \varepsilon_{i} U^{w(\square)-w\left(\square_{i}\right)} \square_{i}
$$

One verifies that $\partial_{U}^{2}=0$. We set $\operatorname{deg} U=-2$ and we introduce the homological grading of a generator by

$$
\operatorname{deg}\left(U^{m} \square\right)=-2 m+\operatorname{dim}(\square)-2 w(\square) .
$$

The differential $\partial_{U}$ decreases the homological grading by 1 .

Remark 4.1.5. It is clear that the weight functions $w(v)$ and $w(v)+$ const define isomorphic lattice complexes. However, the shift of $w$ by a constant induces a shift in the homological degree (4.1.4) as well.

Definition 4.1.6. We define a $\mathbb{Z}^{r}$-indexed filtration on the complex $\mathcal{L}_{w}^{-}$as follows: the subcomplex $\mathcal{L}_{w}^{-}(u)\left(u \in \mathbb{Z}^{r}\right)$ is generated over $\mathbb{Z}[U]$ by all the cubes $\square(v, K)$ with $v \succeq u$.

It is easy to see that $\partial_{U}$ preserves the filtration, so $\mathcal{L}_{w}^{-}(u)$ is a subcomplex of $\mathcal{L}_{w}^{-}$for all $u$. The next theorem shows that the homologies of different subcomplexes, and the homology of $\mathcal{L}_{w}^{-}$ itself, is simple (compatibly with facts from Heegaard Floer link theory, cf. Theorem 2.2.2).

Theorem 4.1.7. Assume that $w$ is non-decreasing. Then the following facts hold.

(a) The homology of $\mathcal{L}_{w}^{-}(u)$ is isomorphic to $\mathbb{Z}[U]$ (as $\mathbb{Z}[U]-$ module). It is generated by the class $\square(u, \emptyset)$ of homological degree $-2 w(u)$.

(b) If additionally $w(v)=w(\max \{0, v\})$, then the inclusion $\mathcal{L}_{w}^{-}(0) \subset \mathcal{L}_{w}^{-}$induces an isomorphism at the level of homology. In particular, the homology of $\mathcal{L}_{w}^{-}$is $\mathbb{Z}[U]$.

Note that the assumptions on $w$ are satisfied by the Hilbert function $h$ of a curve, see (3.1.3).

\footnotetext{
${ }^{2}$ Note that here and below a full square $\mathbf{D}$ denotes a geometric object $\left(|K|\right.$-dimensional solid cube in $\left.\mathbb{R}^{r}\right)$, while a hollow square $\square$ denotes the corresponding abstract generator in a chain complex.
} 
Proof. (a) For every $k \geq w(u)$ let us define the topological space $S_{k}(u):=\bigcup \mathbf{\square}(v, K) \subset \mathbb{R}^{r}$, where the union is over cubes $\square(v, K)$ with $v \succeq u$ and $w(\square(v, K))=w\left(v+e_{K}\right) \leq k$. Note that $\mathbf{\square}(u, \emptyset)$ satisfies the requirements, hence $S_{k}(u)$ is non-empty, it contains $u$.

Similarly to [23, Theorem 3.1.12], we show the following isomorphism of $\mathbb{Z}$-modules for any $q \in \mathbb{Z}$ :

$$
H_{q}\left(\mathcal{L}_{w}^{-}(u)\right)=\bigoplus_{\substack{k \geq w(u) \\ q^{\prime}-2 k=q}} H_{q^{\prime}}\left(S_{k}(u), \mathbb{Z}\right)
$$

This can be proved as follows. Let $\mathcal{C}_{*}\left(S_{k}(u)\right)$ be the usual cubical chain complex of $S_{k}(u)$. $\oplus_{k \geq h(u)} \mathcal{C}_{*}\left(S_{k}(u)\right)$ is their direct sum (as chain complexes), where we prefer to write $(k, \alpha)$ for an element of the $k$-th component. We define the $\mathbb{Z}$-linear morphism $\Phi: \mathcal{L}_{w}^{-}(u) \rightarrow$ $\oplus_{k \geq h(u)} \mathcal{C}_{*}\left(S_{k}(u)\right)$ by $U^{l} \square(v, K) \mapsto\left(l+w\left(v+e_{K}\right), \square(v, K)\right)$, where the latter cube $\square(v, K)$ is considered in $\mathcal{C}_{|K|}\left(S_{k}(u)\right)$, positioned in the component $k=l+w\left(v+e_{K}\right)$. This is a linear isomorphism with inverse $(k, \square(v, K)) \mapsto U^{k-w(\square(v, K))} \square(v, K)$. Moreover, $\Phi\left(\partial_{U}\left(U^{l} \square(v, K)\right)\right)=$ $\partial \Phi\left(U^{l} \square(v, K)\right)$ (where $\partial$ means the direct sum of usual boundary operators of $\mathcal{C}_{*}\left(S_{k}(u)\right)$ ).

Furthermore, multiplication by $U$ in $\mathcal{L}_{w}^{-}(u)$ corresponds to the operator $(k, \square) \mapsto(k+$ $1, i(\square))$, where $i$ is induced by the inclusion $S_{k} \hookrightarrow S_{k+1}$ at the level of $\oplus_{k \geq w(u)} \mathcal{C}_{*}\left(S_{k}(u)\right)$.

Hence, $\Phi$ induces a morphism at the level of homology. If the homological degree $-2 l+$ $|K|-2 w\left(v+e_{K}\right)$ of $U^{l} \square\left(v+e_{K}\right)$ is denoted by $q$, then its homological class is sent by $\Phi_{*}$ into $H_{q^{\prime}}\left(S_{k}\right)$, where $q^{\prime}=|K|$ and $2 k=2\left(l+w\left(v+e_{K}\right)\right)=|K|-q=q^{\prime}-q$. Hence (4.1.8) follows.

Next, we prove that $S_{k}(u)$ is contractible for all $k$. Indeed, since $w$ is non-decreasing, if $\mathbf{\square}(v, K) \subset S_{k}(u)$, then the set $S_{k}(u)$ contains the whole parallelepiped $\left\{x: u \preceq x \preceq v+e_{K}\right\}$. Such a space can be contracted to the lattice point $u$.

In particular, in (4.1.8) $q^{\prime}$ should be zero, $q=-2 k$ and $k \geq w(u)$, while $H_{0}\left(S_{k}(u)\right)=\mathbb{Z}$. This means that $H_{q}\left(\mathcal{L}_{w}^{-}(u)\right)$ is zero unless $q=-2 w(v)-2 l$ for $l \geq 0$, and in this case it is $\mathbb{Z}$ corresponding to the generator $\square(u, \emptyset)$ considered in $S_{w(u)+l}$; or, in the homology of $\mathcal{L}_{w}^{-}(u)$, to the class of $U^{l} \square(u, \emptyset)$. Hence

$$
H_{*}\left(\mathcal{L}_{w}^{-}(u)\right)=\mathbb{Z}[U] \cdot \square(u, \emptyset) .
$$

(b) For $1 \leq p \leq r$ we define the sub-complex $\mathcal{L}_{w, p}^{-}$of $\mathcal{L}_{w}^{-}$generated over $\mathbb{Z}[U]$ by cubes $\square(v, K)$ with $v=\left(v_{1}, \ldots, v_{r}\right), v_{i} \geq 0$ for $1 \leq i \leq p$. Then $\mathcal{L}_{w, r}^{-}=\mathcal{L}_{w}^{-}(0)$ and we also set $\mathcal{L}_{w, 0}^{-}:=\mathcal{L}_{w}^{-}$. We show that $\mathcal{L}_{w, p}^{-} \subset \mathcal{L}_{w, p-1}^{-}$is a homotopy equivalence, hence (b) follows by induction on $p$.

Let $\left(Q_{p-1}, \partial^{Q}\right)$ be the quotient complex $\mathcal{L}_{w, p-1}^{-} / \mathcal{L}_{w, p}^{-}$. It is generated by cubes $\square(v, K)$ with $v_{i} \geq 0$ for $1 \leq i \leq p-1$ and $v_{p}<0$. Note that for such a lattice point one has $w(v)=w(v+$ $\left.e_{p}\right)$. Therefore, $\left(Q_{p-1}, \partial^{Q}\right)$ is a tensor product of two complexes $\left(R_{p-1}, \partial^{R}\right) \otimes\left(T, \partial^{T}\right)$, where $\left(T, \partial^{T}\right)$ is the quotient lattice complex $\mathbb{R} / \mathbb{R}_{\geq 0}$ associated with the constant zero weight (this corresponds to the $p$-th coordinate). More precisely, $T$ is generated by 0 -cubes $a_{n}:=\square(n, \emptyset)$ and 1 -cubes $\alpha_{n}:=\square(n,\{1\})$ for $n \in \mathbb{Z}_{<0}$, and $\partial^{T}\left(\alpha_{n}\right)=a_{n+1}-a_{n}$ (with the notation $a_{0}=0$ ). It is easy to check that the homology of $\left(T, \partial^{T}\right)$ is trivial, hence $H_{*}\left(Q_{p-1}, \partial^{Q}\right)=0$ too.

The point is that the really interesting information is codified in the associated graded versions and in the pages of the corresponding spectral sequences converging to $H_{*}\left(\mathcal{L}_{w}^{-}\right)$.

Definition 4.1.9. We define the multi-graded direct sum complex $\operatorname{gr} \mathcal{L}^{-}=\oplus_{v} \operatorname{gr}_{v} \mathcal{L}^{-}$, where

$$
\operatorname{gr}_{v} \mathcal{L}^{-}=\mathcal{L}^{-}(v) / \sum_{i=1}^{r} \mathcal{L}^{-}\left(v+e_{i}\right)
$$


with induced boundary operator gr $\partial_{U}$. The graded homology group $\mathrm{HL}^{-}=\oplus_{v} \mathrm{HL}^{-}(v)$, where

$$
\mathrm{HL}^{-}(v):=H_{*}\left(\operatorname{gr}_{v} \mathcal{L}^{-}, \operatorname{gr}_{v} \partial_{U}\right),
$$

is called the local lattice homology associated with the weight function $w$. It has an induced $\mathbb{Z}[U]$ module structure.

Remark 4.1.10. Consider the filtration $\left\{F_{n}\right\}_{n \in \mathbb{Z}}$, where the sub-complex $F_{n}$ of $\mathcal{L}_{w}^{-}$is generated over $\mathbb{Z}[U]$ by cubes $\square(v, K)$ with $|v| \geq n$. Then $F_{n} / F_{n+1}=\oplus_{|v|=n} \operatorname{gr}_{v} \mathcal{L}_{w}^{-}$, and $\oplus_{n} F_{n} / F_{n+1}=$ $\operatorname{gr} \mathcal{L}_{w}^{-}$. Therefore, there exists a spectral sequence

$$
E^{1}=H_{*}\left(\operatorname{gr} \mathcal{L}_{w}^{-}\right) \Rightarrow E^{\infty}=H_{*}\left(\mathcal{L}_{w}^{-}\right) .
$$

Remark 4.1.11. The bigrading of $\mathcal{L}_{w}^{-}$. The following bigrading helps to enlighten some hidden structure of the lattice homology (cf. part (3) of Theorem 4.2.1 and the proof after it). We define the following improvement of the homological grading (4.1.4)

$$
\operatorname{bdeg}\left(U^{m} \square\right)=(-2 m-2 w(\square), \operatorname{dim}(\square)) \in \mathbb{Z}^{2} .
$$

Then the boundary operator $\partial_{U}$ has bidegree $(0,-1)$. In particular, $H L^{-}(v)$ is also bigraded. Let $H L_{a, b}^{-}(v)$ denote the corresponding $(a, b)$-component of $H^{-}(v)$.

4.2. The case of algebraic curves. Given a curve singularity $C$ with Hilbert function $h(v)$, one can consider the lattice complex with the weight function $v \mapsto h(v)$ (which is nondecreasing). In this case we will abbreviate the notation to $\mathcal{L}^{-}=\mathcal{L}_{C}^{-}:=\mathcal{L}_{h}^{-}$.

Theorem 4.2.1. (1) Consider the motivic Poincaré series of $C, \mathcal{P}(t ; q)=\sum_{v} \pi_{v}(q) t^{v}$. Then the Poincaré polynomial of $\mathrm{HL}^{-}(v)$, namely $P_{v}^{\mathcal{L}^{-}}(t):=\sum_{i} t^{i} \operatorname{rank} H_{i}\left(\operatorname{gr}_{v} \mathcal{L}^{-}, \operatorname{gr}_{v} \partial_{U}\right)$, satisfies

$$
P_{v}^{\mathcal{L}^{-}}\left(-t^{-1}\right)=t^{h(v)} \cdot \pi_{v}(t) .
$$

In particular, $(-1)^{h(v)} \cdot \pi_{v}(-q)$ is a polynomial in $q$ with non-negative coefficients.

Moreover, the Euler characteristic $P_{v}^{\mathcal{L}^{-}}(-1)=\sum_{i}(-1)^{i} \operatorname{rank} H_{i}\left(\operatorname{gr}_{v} \mathcal{L}^{-}\right)$equals $\pi_{v}(1)=$ $\pi_{v}$, the $v$-coefficient of the Poincaré series.

(2) Furthermore, $H_{-2 h(v)-p}\left(\operatorname{gr}_{v} \mathcal{L}^{-}, \operatorname{gr}_{v} \partial_{U}\right) \simeq H^{p}\left(\mathbb{P} \mathcal{H}^{\prime}(v), \mathbb{Z}\right)$, where $\mathbb{P} \mathcal{H}^{\prime}(v)$ is the complement of the projective hyperplane arrangement defined in 3.6

(3) If $H_{a, b}\left(\operatorname{gr}_{v} \mathcal{L}^{-}, \operatorname{gr}_{v} \partial_{U}\right) \neq 0$ then necessarily $a+2 b=-2 h(v)($ or, $\operatorname{deg}=-2 h(v)-b)$.

(4) The U-action on $H_{*}\left(\operatorname{gr}_{v} \mathcal{L}^{-}, \operatorname{gr}_{v} \partial_{U}\right)$ is trivial.

We postpone the proof of Theorem 4.2.1 till subsection 5.4, where we will use hyperplane arrangements and their Orlik-Solomon algebras. The surprising similarities between the OrlikSolomon complex and the lattice complex will be used deeply. Nevertheless, here we will show how (4.2.2) can be deduced from (3). This also shows that (4.2.2) is not the output of a merely homological manipulation, but it reflects a deeper vanishing property of the Orlik-Solomon algebras.

Proof. (3) $\Rightarrow(4.2 .2)$. For an bigraded $\mathbb{Z}$-module $\left\{H_{a, b}\right\}_{a, b}$ set the virtual Poincaré polynomial $P_{\text {bdeg }}^{\text {vir }}(t):=\sum_{a, b}(-1)^{b} t^{a}$ rank $H_{a, b}$. In particular, this applied to $\operatorname{gr}_{v} \mathcal{L}^{-}$, and counting the bidegrees of the cubes $\left\{U^{m} \square(v, K)\right\}_{m \geq 0, K \subset K_{0}}$, we get

$$
P_{\mathrm{bdeg}}^{v i r}(t)\left(\operatorname{gr}_{v} \mathcal{L}^{-}\right)=\sum_{K \subset K_{0}}(-1)^{|K|} \cdot \frac{t^{-2 h\left(v+e_{K}\right)}}{1-t^{-2}}=\pi_{v}\left(t^{-2}\right) .
$$


Since the differential $\partial_{U}$ has bi-degree $(0,-1)$, the virtual Poincaré polynomials of the complex and its homology coincide and we get $P_{\mathrm{bdeg}}^{v i r}(t)\left(H F^{-}(v)\right)=P_{\mathrm{bdeg}}^{v i r}(t)\left(\operatorname{gr}_{v} \mathcal{L}^{-}\right)$, hence

$$
\sum_{a, K}(-1)^{|K|} t^{a} \cdot \operatorname{rank} H L_{a,|K|}^{-}(v)=\pi_{v}\left(t^{-2}\right) .
$$

Then (4.2.2) is equivalent to

$$
\sum_{a, K}(-1)^{|K|} t^{a} \cdot \operatorname{rank} H L_{a,|K|}^{-}(v)=\sum_{a, K}(-1)^{|K|} t^{2 a+2 h(v)+2|K|} \cdot \operatorname{rank} H L_{a,|K|}^{-}(v) .
$$

But this is true, since $a=2 a+2 h(v)+2|K|$ whenever $H L_{a,|K|}^{-}(v) \neq 0$ by (3).

Corollary 4.2.4. $v \in \mathcal{S}$ if and only if $\mathrm{HL}^{-}(v) \neq 0$. For any $v \in \mathcal{S}$ one has $P_{v}^{\mathcal{L}^{-}}\left(-t^{-1}\right)=$ $t^{2 h(v)}+$ higher order terms. (This shows that the class of $\square(v, \emptyset)$ does not vanish in $\mathrm{HL}^{-}(v)$.) In particular, $P_{v}^{\mathcal{L}^{-}}(t)$ and $\pi_{v}(q)$ determine each other.

Proof. Use Lemma 3.7.3 (and its proof) and the identity (4.2.2).

\subsection{Example. The case of a curve with one component.}

Suppose that $r=1$. We will abbreviate $\square(v, \emptyset)=a_{v}, \square(v,\{1\})=\alpha_{v}$. If $v \notin \mathcal{S}$ then $\left(\operatorname{gr}_{v} \partial_{U}\right)\left(\alpha_{v}\right)=a_{v}$, hence HL $\mathrm{HL}^{-}(v)=0$. If $v \in \mathcal{S}$ then $\left(\operatorname{gr}_{v} \partial_{U}\right)\left(\alpha_{v}\right)=U a_{v}$, hence $\mathrm{HL}^{-}(v)=$ $\mathbb{Z}\left\langle a_{v}\right\rangle$ of homological degree $-2 h(v)$. Hence for $v \in \mathcal{S}$ one has $P_{v}^{\mathcal{L}^{-}}(t)=t^{-2 h(v)}$ compatibly with $\mathcal{P}(t ; q)=\sum_{v \in \mathcal{S}} q^{h(v)} t^{v}$.

Furthermore, the spectral sequence from Remark 4.1.10 satisfies $E^{1} \simeq E^{\infty} \simeq \mathbb{Z}[U]$ as $\mathbb{Z}$ modules. Nevertheless, $E^{1} \not E^{\infty}$ as $\mathbb{Z}[U]$ modules: $E^{1}$ has trivial $U$-action, while in $E^{\infty}$ the $U$-action sends the generator of a semigroup element to the generator of the consecutive semigroup element.

Remark 4.3.1. (The $U=0$ (or "hat"-) version.) (a) It is interesting to consider the complex $\mathcal{L}_{U=0}^{-}$too (obtained from $\mathcal{L}^{-}$via substitution $U=0$ ), generated over $\mathbb{Z}$ by the cubes and boundary operator given by (4.1.3) with substitution $U=0$. Then $H_{*}\left(\mathcal{L}_{U=0}^{-}\right)=\mathbb{Z}$ (generated by the class of $\left.a_{0}\right)$. Moreover, the filtration $F_{n}^{\prime}:=\left.F_{n}\right|_{U=0}$ induces a spectral sequence $\left\{E_{U=0}^{k}\right\}_{k} . F_{n}^{\prime} / F_{n+1}^{\prime}$ is generated over $\mathbb{Z}$ by all $a_{v}$ and $\alpha_{v}$, and the only non-trivial components of the boundary map are the isomorphisms $\mathbb{Z}\left\langle\alpha_{v}\right\rangle \rightarrow \mathbb{Z}\left\langle a_{v}\right\rangle$ for any $v \notin \mathcal{S}$. Hence $E_{U=0}^{1}$ is $\oplus_{v \in \mathcal{S}} \mathbb{Z}\left\langle a_{v}, \alpha_{v}\right\rangle$ of homological degrees $-2 h(v)$ and $-2 h(v)-1$ repectively. The non-trivial components of the $d^{1}: E_{U=0}^{1} \rightarrow E_{U=0}^{1}$ operator are the isomorphisms $\mathbb{Z}\left\langle\alpha_{v}\right\rangle \rightarrow \mathbb{Z}\left\langle a_{v+1}\right\rangle$ whenever both $v$ and $v+1$ are elements of $\mathcal{S}$. Hence, the $E_{U=0}^{2}$ term is

$$
E_{U=0}^{2}(v)= \begin{cases}\mathbb{Z}\left\langle a_{v}, \alpha_{v}\right\rangle & \text { if } v \in \mathcal{S}, v-1 \notin \mathcal{S}, v+1 \notin \mathcal{S}, \\ \mathbb{Z}\left\langle a_{v}\right\rangle & \text { if } v \in \mathcal{S}, v-1 \notin \mathcal{S}, v+1 \in \mathcal{S}, \\ \mathbb{Z}\left\langle\alpha_{v}\right\rangle & \text { if } v \in \mathcal{S}, v-1 \in \mathcal{S}, v+1 \notin \mathcal{S}, \\ 0 & \text { otherwise. }\end{cases}
$$

The parity of the homological degree provides a $\mathbb{Z}_{2}$ grading $\left\{E_{U=0}^{2}\right\}_{\epsilon}$ of $E_{U=0}^{2}$, where $\epsilon \in\{0,1\}$ has the same parity as the homological degree. Then, since $\Delta(t)=(1-t) \sum_{v \in \mathcal{S}} t^{v}$,

$$
\sum_{v, \epsilon}(-1)^{\epsilon} \operatorname{rank}\left(E_{U=0}^{2}(v)_{\epsilon}\right) t^{v+\epsilon}=\Delta(t) .
$$

Since for irreducible plane curves $\mathcal{S}$ and $\Delta$ classifies the topological type of the knot of $C$, cf. [36], both $E_{U=0}^{1}$ and $E_{U=0}^{2}$ terms contain the complete information about the local topological type of $C$. Note also that $E_{U=0}^{2}$ is supported in $[0, \mu]$, where $\mu=2 \delta$ is the Milnor number of $C$, and $v \mapsto \mu-v-2 \epsilon$ is a symmetry of $E_{U=0, \epsilon}^{2}$ which preserves the $\epsilon$-degree. 
The $E_{U=0}^{\infty}$ term is $H_{*}\left(\mathcal{L}_{U=0}^{-}\right)=\mathbb{Z}$.

(b) The short exact sequence of complexes $0 \rightarrow \mathcal{L}^{-} \stackrel{U}{\longrightarrow} \mathcal{L}^{-} \rightarrow \mathcal{L}_{U=0}^{-} \rightarrow 0$ induces a long exact sequence connecting the groups $H L^{-}(v)$ with the ' $U=0$ '-counterparts, whose explicit description is left to the reader.

\subsection{Example. The case of a curve with two components.}

We will abbreviate $\square(v, \emptyset)=a_{v}, \square(v,\{1\})=\alpha_{v} \square(v,\{2\})=\beta_{v}$ and $\square(v,\{1,2\})=\Gamma_{v}$. By the general theory, if $v \notin \mathcal{S}$ then $H L^{-}(v)=0$. If $v \in \mathcal{S}$ there are two cases.

a) $h(v)=h\left(v+e_{1}+e_{2}\right)-1 ; \alpha_{v} \mapsto U a_{v}, \beta_{v} \mapsto U a_{v}, \Gamma_{v} \mapsto \alpha_{v}-\beta_{v}$, then $\mathrm{HL}^{-}(v)=\mathbb{Z}\left\langle a_{v}\right\rangle$ of homological degree $-2 h(v)$. (In this case, $\mathbb{P H}(v)=$ point.)

b) $h(v)=h\left(v+e_{1}+e_{2}\right)-2 ; \alpha_{v} \mapsto U a_{v}, \beta_{v} \mapsto U a_{v}, \Gamma_{v} \mapsto U \alpha_{v}-U \beta_{v}$, then $\mathrm{HL}^{-}(v)=$ $\mathbb{Z}\left\langle a_{v}, \alpha_{v}-\beta_{v}\right\rangle$ of homological degrees $-2 h(v),-1-2 h(v)$. (Cf. with $\mathbb{P} \mathcal{H}(v)=\mathbb{P}^{1} \backslash 2$ points.)

In case (b) the Euler characteristic of $\mathrm{HL}^{-}(v)$ (and the corresponding coefficient in the Alexander polynomial) vanishes, but the homology and the coefficient in the motivic Poincaré series do not vanish. This case appears, for example, for all $v$ in the conductor of $C$.

Using Figures 11 and 2 , one can compute the $\mathrm{HL}^{-}$for the singularities of types $A_{3}$ and $D_{5}$. The analogous computation for the two-component singularity $A_{2 n-1}$ agrees with the computations of the Heegaard Floer link homology in [29]. An explicit computation in the case $A_{1}$ is given in section 7

\subsection{Application to the theory of deformations of singularities.}

In this subsection we consider deformations of plane curve singularities. ¿From topological point of view, they induce cobordisms between the corresponding links in the three-sphere, hence maps between their Heegaard Floer link homologies. We present here the analogous maps in lattice homology, under the restriction that the central fiber of the deformation is irreducible (while the generic fiber is allowed to have several components).

We wish to emphasize that semicontinuity results for different singularity invariants are crucial in the deformation theory of singularities, since they might provide more information about the (open) problem of adjacencies of singularity types.

Proposition 4.5.1. Let $\left(C^{\prime}, 0\right)$ be a curve singularity with $r$ irreducible components, and assume that it is a deformation of an irreducible germ $(C, 0)$. Then $h_{C^{\prime}}(v) \geq h_{C}(|v|)$ for every $v \in \mathbb{Z}^{r}$.

Proof. 3 By Corollary 3.4.7 the Hilbert function is determined by the topological type of a singularity. Consider the family of curves $C_{t}$ with the central fiber $C_{0}=C$ and the generic fiber $C_{t}$ topologically equivalent to $C^{\prime}$. Let us fix $v \in \mathbb{Z}^{r}$. One can assume that $h_{C_{t}}(v)$ is constant for small enough (but nonzero) $t$.

We get a family of subspaces $J_{C_{t}}(v)$ in $\mathcal{O}$ (or rather in a sufficiently large jet space $j_{N} \mathcal{O}$ ) of fixed codimension $h_{C_{t}}(v)=h_{C^{\prime}}(v)$. Since the Grassmannian $\operatorname{Gr}\left(h_{C^{\prime}}(v), j_{N} \mathcal{O}\right)$ is compact, this family has a well defined limit $J_{0}(v)=\lim _{t \rightarrow 0} J_{C_{t}}(v)$.

Let us prove the inclusion $J_{0}(v) \subset J_{C}(|v|)$. Indeed, every function $g$ in this limiting subspace is a limit of a sequence of functions $g_{t}$ intersecting $C_{t}$ with multiplicity at least $|v|$, so by the semicontinuinty of the intersection multiplicity $g$ should intersect $C$ with multiplicity at least $|v|$ too. Therefore $J_{0}(v) \subset J_{C}(|v|)$ and $h_{C}(|v|)=\operatorname{codim} J_{C}(|v|) \leq \operatorname{codim} J_{0}(v)=h_{C^{\prime}}(v)$.

\footnotetext{
${ }^{3}$ We thank Maria Pe Pereira and Patrick Popescu-Pampu for noting a gap in the first version of the proof of this proposition.
} 
After the first version of this paper appeared on arXiv, Borodzik and Livingston [2] gave an alternative proof of this proposition (only for $\delta$-constant deformations) using Heegaard Floer theory.

Theorem 4.5.2. Suppose that a (possibly reducible) curve $C^{\prime}$ is a deformation of an irreducible curve $C$. Then there exists a natural chain map $\phi: \mathcal{L}_{C^{\prime}}^{-} \rightarrow \mathcal{L}_{C}^{-}$, with $\phi(\square(v, \emptyset))=$ $U^{h_{C^{\prime}}(v)-h_{C}(|v|)} \square(|v|, \emptyset)$ and $\phi\left(\mathcal{L}_{C^{\prime}}^{-}(v)\right) \subset \mathcal{L}_{C}^{-}(|v|)$ for any $v \in \mathbb{Z}^{r}$. Moreover, for any $v$, the induced map

$$
\phi_{*}(v): H_{*}\left(\mathcal{L}_{C^{\prime}}^{-}(v)\right) \rightarrow H_{*}\left(\mathcal{L}_{C}^{-}(|v|)\right)
$$

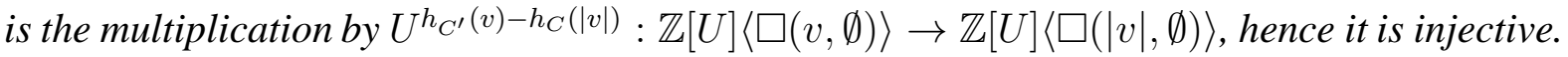

Proof. Let us define a map $\phi$ acting on the generators of the lattice complex as follows. For a 0 - or a 1 - dimensional cube in $\mathbb{Z}^{r}$ one can define its natural projection onto $\mathbb{Z}$ by

$$
p(\square(v, \emptyset)):=\square(|v|, \emptyset) ; \quad p(\square(v,\{i\})):=\square(|v|, 1) .
$$

Then for an arbitrary cube $\square$ define

$$
\phi(\square):= \begin{cases}U^{h_{C^{\prime}}(\square)-h_{C}(p(\square))} p(\square) & \text { if } \operatorname{dim} \square \leq 1, \\ 0 & \text { if } \operatorname{dim} \square>1 .\end{cases}
$$

By Lemma 4.5.1 the power of $U$ above is nonnegative, hence $\phi$ is well-defined. It preserves the filtration on $\mathcal{L}^{-}$and it commutes with the differentials (by a straightforward computation left to the reader). The injectivity of $\phi_{*}(v)$ follows from Theorem 4.1.7.

We plan to study deformation theoretical applications in more details in the future.

\section{CENTRAL HyPERPLANE ARRANGEMENTS}

\subsection{Matroids and rank functions.}

Definition 5.1.1. (a) ([34] $)$ Let $K_{0}$ be a finite set. A function $\rho$, assigning a non-negative integer to any subset $K \subset K_{0}$, is called a rank function, if

(1) $0 \leq \rho(K) \leq|K|$.

(2) If $K_{1} \subset K_{2}$ then $\rho\left(K_{1}\right) \leq \rho\left(K_{2}\right)$.

(3) For every pair of subsets $K_{1}$ and $K_{2}$ one has

$$
\rho\left(K_{1} \cap K_{2}\right)+\rho\left(K_{1} \cup K_{2}\right) \leq \rho\left(K_{1}\right)+\rho\left(K_{2}\right) .
$$

(b) A matroid $M=\left(K_{0}, \rho\right)$ is a finite set $K_{0}$ with a rank function $\rho$ defined on it.

(c) The characteristic polynomial of a matroid $M=\left(K_{0}, \rho\right)$ is defined as

$$
\chi_{M}(t)=\sum_{K \subset K_{0}}(-1)^{|K|} t^{\rho\left(K_{0}\right)-\rho(K)} .
$$

Remark 5.1.2. Some authors define the characteristic polynomial using the Möbius function of a matroid. This definition is equivalent to the present one, see e.g. [34, Theorem 2.4].

Let $h(v)$ denote the Hilbert function of a plane curve singularity. Let us fix $K_{0}=\{1, \ldots, r\}$ and for every $v$ consider the following function on subsets of $K_{0}$ :

$$
\rho_{v}(K):=h\left(v+e_{K}\right)-h(v)=\operatorname{dim} J(v) / J\left(v+e_{K}\right) .
$$

Then Lemmas 3.5.1 and 3.5.2 show that for every $v$ the function $\rho_{v}$ is a rank function on $K_{0}$.

We will call $\rho_{v}$ the rank function for a the local matroid $M_{v}$. In the space $J(v)$ we have $r$ subspaces $J\left(v+e_{i}\right)$ of codimension 0 or 1 . If $v \in \mathcal{S}$, then the set of functions with valuation 
$v$ can be represented as a complement of a hyperplane arrangement (cf. [18], or 3.6 here). If $v \notin \mathcal{S}$, then $J(v)=J\left(v+e_{i}\right)$ for some $i$ (cf. Lemma 3.5.1), hence $J\left(v+e_{K}\right)=J\left(v+e_{K}+e_{i}\right)$ for any $K$ with $K \not \supset i$ by 3.5.2. Therefore, in this case, by pairwise cancelation, $\chi_{M_{v}}(t)=0$.

5.2. Some general facts on central hyperplane arrangements. Let $V$ be a vector space and let $\mathcal{H}=\left\{\mathcal{H}_{1}, \ldots, \mathcal{H}_{r}\right\}$ be a collection of linear hyperplanes in $V$. For a subset $K$ of $K_{0}=\{1, \ldots, r\}$ we define $\rho(K)=\operatorname{codim} \cap_{i \in K} \mathcal{H}_{i}$. One can check that $\rho$ is a rank function on $K_{0}$. Let us denote by $\chi_{\mathcal{H}}(t)$ its characteristic polynomial.

To an arrangement $\mathcal{H}$ one associates the corresponding Orlik-Solomon algebra as follows. Consider the anticommutative algebra $\mathcal{E}$ generated by the variables $z_{1}, \ldots, z_{r}$ corresponding to hyperplanes. For any set $K=\left\{i_{1}, \ldots, i_{k}\right\} \subset K_{0}$ we consider the monomial $z_{K}=z_{i_{1}} \wedge \cdots \wedge$ $z_{i_{k}} \in \mathcal{E}$. We can equip $\mathcal{E}$ with the natural differential $\partial$ sending $z_{i}$ to 1 , namely

$$
\partial\left(z_{K}\right)=\sum_{j=1}^{k}(-1)^{j-1} z_{K \backslash\left\{i_{j}\right\}} .
$$

The natural degree of $z_{K}$ is $|K|$. Hence $\partial$ has degree -1 .

Definition 5.2.1. We call the set $K$ dependent, if the linear equations of the corresponding hyperplanes are linearly dependent. Otherwise $K$ is called independent.

The Orlik-Solomon ideal $\mathcal{I}$ is the ideal in $\mathcal{E}$ generated by the elements $\partial z_{K}$ for all dependent sets $K$. The Orlik-Solomon algebra is the quotient $\mathcal{A}=\mathcal{E} / \mathcal{I}$.

Theorem 5.2.2. ([24, Theorem 5.2]) The integral cohomology ring of the complement $V$ \ $\cup_{i=1}^{r} \mathcal{H}_{i}$ is isomorphic to the Orlik-Solomon algebra $\mathcal{E} / \mathcal{I}$. It has no torsion, and its Poincaré polynomial is given by the formula

$$
P(\mathcal{H}, t)=(-t)^{\rho\left(K_{0}\right)} \cdot \chi_{\mathcal{H}}\left(-t^{-1}\right)=\sum_{K \subset K_{0}}(-1)^{|K|}(-t)^{\rho(K)} .
$$

As a corollary, we conclude that the homology of $V \backslash \cup_{i=1}^{r} \mathcal{H}_{i}$ is defined by its class in the Grothendieck ring, cf. Lemma 3.7.1. The same is true for its projectivization (see below). (This property of hyperplane complements explain why the coefficients of the motivic Poincare series can guide the complete cohomological information.)

Later we will define a distinguished homological degree in $\mathcal{E}$, such that the above isomorphism will preserve the corresponding gradings.

First, we consider the following 'deformation of the differential on $\mathcal{E}$ '.

Definition 5.2.3. Let us define the following operator:

$$
\partial_{U}: \mathcal{E}[U] \rightarrow \mathcal{E}[U], \quad \partial_{U}\left(z_{K}\right)=\sum_{j=1}^{k}(-1)^{j-1} U^{\rho(K)-\rho\left(K \backslash\left\{i_{j}\right\}\right)} z_{K \backslash i_{j}},
$$

where $U$ is a formal variable and $K=\left\{i_{1}, \ldots, i_{k}\right\}$.

Note that $\rho(K)-\rho\left(K \backslash\left\{i_{j}\right\}\right) \in\{0,1\}$, hence $\partial_{U}$ decomposes into a sum of two components

$$
\partial_{U}=\partial_{0}+U \partial_{1}, \quad \text { with } \partial_{0}+\partial_{1}=\partial .
$$

Lemma 5.2.5. The operator $\partial_{U}$ is a differential on $\mathcal{E}[U]$, that is, $\partial_{U}^{2}=0$. In particular, the following identities hold:

$$
\partial_{0}^{2}=\partial_{1}^{2}=0, \quad \partial_{0} \partial_{1}+\partial_{1} \partial_{0}=0
$$

Proof. Straightforward. 
Let $\mathcal{J}$ and $\mathcal{J}^{\perp}$ denote the subspaces of $\mathcal{E}$ spanned by the elements $z_{K}$ for all dependent, respectively independent subsets $K$. Clearly $\mathcal{E}=\mathcal{J} \oplus \mathcal{J}^{\perp}$.

Lemma 5.2.6. The following statements hold:

(a) ([24, Lemma 2.7], [25, Lemma 3.15]) $\mathcal{I}=\mathcal{J}+\partial \mathcal{J}$.

(b) $\partial_{0} \mathcal{J}^{\perp}=0$, hence $\operatorname{Im} \partial_{0}=\partial_{0} \mathcal{J}$.

(c) $\partial_{1} \mathcal{J} \subset \mathcal{J}$, hence $\mathcal{I}=\mathcal{J}+\partial \mathcal{J}=\mathcal{J}+\partial_{0} \mathcal{J}$.

(d) $\operatorname{ker} \partial_{0}=\mathcal{J}^{\perp}+\operatorname{Im} \partial_{0}$.

(e) There exist subspaces $A \subset \mathcal{J}, B \subset \mathcal{J}^{\perp}$ such that $\operatorname{Im} \partial_{0}=A \oplus B$.

Proof. The claims (b) and (c) are clear. Let us prove (d). The inclusion $\mathcal{J}^{\perp}+\operatorname{Im} \partial_{0} \subset \operatorname{ker} \partial_{0}$ is also clear, hence we need to prove that if $\partial_{0}(\phi)=0$ then there exists $\widetilde{\phi} \in \mathcal{J}^{\perp}$ such that $\phi-\widetilde{\phi} \in \operatorname{Im}\left(\partial_{0}\right)$.

Let us call $z_{i}$ essential in a monomial $z_{i} \wedge z_{K}$, if $\rho(\{i\} \sqcup K)=\rho(K)+1$, and redundant otherwise. Let us decompose $\phi=z_{1} \wedge \phi_{1}+z_{1} \wedge \phi_{2}+\phi_{3}$, where $z_{1}$ is essential in every monomial of $z_{1} \wedge \phi_{1}$, redundant in every monomial of $z_{1} \wedge \phi_{2}$, and $\phi_{3}$ contains no $z_{1}$. Then

$$
0=\partial_{0}(\phi)=z_{1} \wedge \psi+\phi_{2}+\partial_{0}\left(\phi_{3}\right)
$$

for some $\psi$, and neither $\phi_{2}$ nor $\partial_{0}\left(\phi_{3}\right)$ contain $z_{1}$. Hence $\phi_{2}=-\partial_{0}\left(\phi_{3}\right)$. Since $z_{1}$ is redundant in every monomial in $z_{1} \wedge \partial_{0}\left(\phi_{3}\right)$, it is redundant in every monomial in $z_{1} \wedge \phi_{3}$ too. Therefore

$$
\partial_{0}\left(z_{1} \wedge \phi_{3}\right)=\phi_{3}-z_{1} \wedge \partial_{0}\left(\phi_{3}\right)+z_{1} \wedge \eta
$$

where $z_{1}$ is essential in every monomial of $z_{1} \wedge \eta$. Indeed, if $i_{j} \in K, z_{i_{j}}$ is redundant in $K \cup\{1\}$ and essential in $K$, then $z_{1}$ is essential in $K \cup\{1\} \backslash\left\{i_{j}\right\}$. We conclude that

$$
\phi-\partial_{0}\left(z_{1} \wedge \phi_{3}\right)=z_{1} \wedge\left(\phi_{1}-\eta\right)
$$

and $z_{1}$ is essential in every monomial in the right hand side. Now, $0=\partial_{0}(\phi)=-z_{1} \wedge \partial_{0}\left(\phi_{1}-\eta\right)$, hence $\partial_{0}\left(\phi_{1}-\eta\right)=0$. Then we can repeat the procedure inductively replacing $\phi$ by $\phi_{1}-\eta$, and $z_{1}$ by $z_{2}$, etc. At the end we reduce $\phi$ modulo $\operatorname{Im}\left(\partial_{0}\right)$ to an element of $\mathcal{E}$ where all $z_{i}$ are essential; such an element belongs to $\mathcal{J}^{\perp}$.

Next, we prove (e). Recall that $\operatorname{Im} \partial_{0}=\partial_{0} \mathcal{J}$ and $K$ is dependent iff $\rho(K)<|K|$. If the monomial $z_{K^{\prime}}$ appears in $\partial_{0}\left(z_{K}\right)$ then $\rho(K)=\rho\left(K^{\prime}\right)$ and $\left|K^{\prime}\right|=|K|-1$. Therefore, with $K$ dependent, $\partial_{0}\left(z_{K}\right) \in \mathcal{J}^{\perp}$ if $\rho(K)=|K|-1$, and $\partial_{0}\left(z_{K}\right) \in \mathcal{J}$ otherwise.

Lemma 5.2.7. (cf. [25, Lemma 3.42], [8, 1.46]) Let $\partial_{1}^{\mathcal{A}}$ be the differential induced by $\partial_{1}$ on $\mathcal{A}=\mathcal{E} / \mathcal{I}$. Then $\partial_{1}^{\mathcal{A}}$ is acyclic, that is, $\operatorname{im} \partial_{1}^{\mathcal{A}}=\operatorname{ker} \partial_{1}^{\mathcal{A}}$.

Proof. In the proof we always refer to the points (a)-(e) of Lemma 5.2.6. Suppose that the class $[\alpha] \in \mathcal{A}=\mathcal{E} / \mathcal{I}$ belongs to the kernel of $\partial_{1}^{\mathcal{A}}$, so $\partial_{1}(\alpha) \in \mathcal{I}$. By (c) we can assume that $\alpha \in \mathcal{J}^{\perp}$. Then $\partial(\alpha)=\partial_{1}(\alpha) \in \mathcal{I} \cap \mathcal{J}^{\perp}$. By (c)-(e) $\mathcal{I} \cap \mathcal{J}^{\perp}=(\mathcal{J}+A \oplus B) \cap \mathcal{J}^{\perp}=B \subset \partial_{0} \mathcal{J}$, hence there exists $\alpha_{1} \in \mathcal{J}$ such that $\partial_{1}(\alpha)=\partial_{0}\left(\alpha_{1}\right)$. Furthermore, $\partial_{0} \partial_{1}\left(\alpha_{1}\right)=\partial_{1} \partial_{0}\left(\alpha_{1}\right)=0$, hence $\partial_{1}\left(\alpha_{1}\right) \in$ ker $\partial_{0} \cap \partial_{1} \mathcal{J}$. But again by (c)-(d)-(e) one has ker $\partial_{0} \cap \partial_{1} \mathcal{J} \subset\left(\mathcal{J}^{\perp}+A \oplus B\right) \cap \mathcal{J}=$ $A \subset \partial_{0} \mathcal{J}$. Hence there exists $\alpha_{2} \in \mathcal{J}$ with $\partial_{1}\left(\alpha_{1}\right)=\partial_{0}\left(\alpha_{2}\right)$. Again, $\partial_{1}\left(\alpha_{2}\right) \in \operatorname{ker} \partial_{0} \cap \partial_{1} \mathcal{J}$. This procedure can be repeated to provide $\alpha_{3} \in \mathcal{J}$ with $\partial_{1}\left(\alpha_{2}\right)=\partial_{0}\left(\alpha_{3}\right)$, and, in fact, a sequence $\alpha_{i} \in \mathcal{J}$ with $\partial_{0}\left(\alpha_{i}\right)=\partial_{1}\left(\alpha_{i-1}\right)\left(\alpha_{0}=\alpha\right)$.

Note that $\rho\left(\alpha_{i}\right)=\rho(\alpha)-i$, so this process eventually stops. Now

$$
\partial\left(\alpha-\alpha_{1}+\alpha_{2}-\ldots\right)=\partial_{1}(\alpha)-\partial_{0}\left(\alpha_{1}\right)-\partial_{1}\left(\alpha_{1}\right)+\partial_{0}\left(\alpha_{2}\right)+\partial_{1}\left(\alpha_{2}\right)-\ldots=0 .
$$

Since $\partial$ is acyclic on $\mathcal{E}$, there exists $\beta$ such that $\partial(\beta)=\alpha-\alpha_{1}+\alpha_{2}-\ldots$. Let us decompose $\beta=\beta^{\prime}+\beta^{\prime \prime}$, where $\beta^{\prime} \in \mathcal{J}^{\perp}$ and $\beta^{\prime \prime} \in \mathcal{J}$, then by (b),

$$
\alpha=\partial_{1}\left(\beta^{\prime}\right)+\partial\left(\beta^{\prime \prime}\right)+\alpha_{1}-\alpha_{2}+\ldots \equiv \partial_{1}\left(\beta^{\prime}\right) \bmod \mathcal{I},
$$


hence $[\alpha]$ belongs to the image of $\partial_{1}^{\mathcal{A}}$.

The following theorem determine the homology of the complexes $\left(\mathcal{E}, \partial_{0}\right)$ and $\left(\mathcal{E}[U], \partial_{U}\right)$.

Theorem 5.2.8. (1) The homology of the differential $\partial_{0}$ is isomorphic to the Orlik-Solomon algebra $\mathcal{A}=\mathcal{E} / \mathcal{I}$. This fact together with Theorem 5.2.2 provide

$$
H_{*}\left(\mathcal{E}, \partial_{0}\right) \simeq \mathcal{A} \simeq H^{*}\left(V \backslash \cup_{i=1}^{r} \mathcal{H}_{i}\right)
$$

(2) The homology of the differential $\partial_{U}$ is isomorphic (as $\mathbb{Z}$-module) to the homology of the projectivized arrangement:

$$
H_{*}\left(\mathcal{E}[U], \partial_{U}\right) \simeq \operatorname{ker} \partial_{1}^{\mathcal{A}} \simeq H^{*}\left(\mathbb{P} V \backslash \cup_{i=1}^{r} \mathbb{P} \mathcal{H}_{i}\right),
$$

and it can be generated by a set of elements of type $U^{m} z_{K}$, with $m=0$ and $K$ independent.

In particular, the induced $U$-action on $H_{*}\left(\mathcal{E}[U], \partial_{U}\right)$ is trivial.

(3) $\mathcal{E}$ is bi-graded: one can assign $|K|$, respectively $\rho(K)$, to $z_{K} . \partial_{0}$ decreases the first grading by 1 and preserves the second one, hence $H_{*}\left(\mathcal{E}, \partial_{0}\right)$ is bi-graded too. Nevertheless, the two gradings on $H_{*}\left(\mathcal{E}, \partial_{0}\right)$ agree, and the isomorphisms from (1) and (3) are graded isomorphisms (where $H^{*}\left(V \backslash \cup_{i=1}^{r} \mathcal{H}_{i}\right)$ and $H^{*}\left(\mathbb{P} V \backslash \cup_{i=1}^{r} \mathbb{P} \mathcal{H}_{i}\right)$ have their natural cohomological gradings).

Proof. (1) By Lemma 5.2.6 one has ker $\partial_{0}=\mathcal{J}^{\perp}+\operatorname{Im} \partial_{0}$ and $\operatorname{Im} \partial_{0}=\partial_{0} \mathcal{J}$, hence

$$
H_{*}\left(\mathcal{E}, \partial_{0}\right)=\left(\mathcal{J}^{\perp}+\partial_{0} \mathcal{J}\right) / \partial_{0} \mathcal{J} \simeq \mathcal{J}^{\perp} /\left(\partial_{0} \mathcal{J} \cap \mathcal{J}^{\perp}\right) \simeq \mathcal{E} /\left(\mathcal{J}+\partial_{0} \mathcal{J}\right)
$$

The last identity follows from the splitting in Lemma5.2.6(e). Then use Lemma5.2.6(c).

(2) Since $\partial_{U}=\partial_{0}+U \partial_{1}$, there exists a spectral sequence starting with $H_{*}\left(\mathcal{E}[U], \partial_{0}\right)$ and converging to $H_{*}\left(\mathcal{E}[U], \partial_{U}\right)$. The $E^{1}$ page is $\left(\left(H_{*}\left(\mathcal{E}[U], \partial_{0}\right), U \partial_{1}^{\mathcal{A}}\right)\right)=\left(\mathcal{A}[U], U \partial_{1}^{\mathcal{A}}\right)$, and by Lemma 5.2.7 the $E^{2}$ page has a form:

$$
H_{*}\left(\mathcal{E}[U], \partial_{U}\right) \simeq H_{*}\left(\mathcal{A}[U], U \partial_{1}^{\mathcal{A}}\right) \simeq \operatorname{ker} \partial_{1}^{\mathcal{A}}
$$

Since this homology is concentrated in the lowest $U$-degree, all higher differentials vanish. This shows the first isomorphism of (2). For the second one see [8, Theorem 1.50].

(3) ¿From the proof of part (1) follows that $H^{*}\left(\mathcal{E}, \partial_{0}\right)$ can be identified with a quotient of $\mathcal{J}^{\perp}$. Since $\mathcal{J}^{\perp}$ is spanned by the independent monomials, the gradings induced by $|K|$ and $\rho(K)$ coincide on $H^{*}\left(\mathcal{E}, \partial_{0}\right)$. The isomorphisms from the already cited [24, Theorem 5.2] and [8, Theorem 1.50] are compatible with this grading.

Remark 5.2.9. (Cf. [25, Corollary 3.58]) Since $V \backslash \cup_{i=1}^{r} \mathcal{H}_{i}=\mathbb{C}^{*} \times\left(\mathbb{P} V \backslash \cup_{i=1}^{r} \mathbb{P} \mathcal{H}_{i}\right)$, the Poincaré polynomials $P(\mathcal{H}, t)$ and $P(\mathbb{P H}, t)$ of the cohomologies of the complements of the linear and projective arrangements satisfies $(1+t) \cdot P(\mathbb{P} \mathcal{H}, t)=P(\mathcal{H}, t)$.

Example 5.2.10. Consider the arrangement of $r$ lines through the origin in $V=\mathbb{C}^{2}$. Then

$$
\begin{gathered}
\partial_{U}(1)=0, \partial_{U}\left(z_{i}\right)=U, \partial_{U}\left(z_{i} \wedge z_{j}\right)=U\left(z_{i}-z_{j}\right)=U \partial\left(z_{i} \wedge z_{j}\right), \\
\partial_{U}\left(z_{K}\right)=\partial\left(z_{K}\right) \text { for }|K| \geq 3 .
\end{gathered}
$$

The homology of $\partial_{U}$ is spanned by $1, z_{1}-z_{2}, \ldots, z_{1}-z_{r}$. On the other hand, $\mathbb{P} V \backslash \mathbb{P H}$ is the complement to $r$ points in $\mathbb{C P}^{1}$, homotopically equivalent to the bouquet of $(r-1)$ circles. 


\subsection{The Orlik-Solomon complex and the lattice complex for curve singularities.}

Consider a curve singularity $C$, the associated lattice complex (cf. Section 4) and the collection of local hyperplane arrangements $\mathcal{H}(v)$ (cf. 3.6). We wish to compare the Orlik-Solomon complex $\left(\mathcal{E}[U], \partial_{U}\right)$ associated with the local hyperplane arrangement $\mathcal{H}(v)$ and the lattice complex $\left(\operatorname{gr}_{v} \mathcal{L}^{-}, \operatorname{gr}_{v} \partial_{U}\right)$.

Theorem 5.3.1. (a) For any fixed $v$ one has an isomorphism

$$
H_{-2 h(v)-b}\left(\operatorname{gr}_{v} \mathcal{L}^{-}, \operatorname{gr}_{v} \partial_{U}\right)=H_{b}\left(\mathcal{E}[U], \partial_{U}\right) .
$$

In the left hand side the homological degree is the one defined in (4.1.4), while in the right hand side is induced by $\operatorname{deg}\left(z_{K}\right)=|K|, c f$. [5.2. (This is a $\mathbb{Z}$ module isomorphism; since $U$ acts on $H_{*}\left(\mathcal{E}[U], \partial_{U}\right)$ trivially, $c f$. 5.2.8 it acts on $H_{*}\left(\operatorname{gr}_{v} \mathcal{L}^{-}, \operatorname{gr}_{v} \partial_{U}\right)$ trivially as well. $)$

(b) Assume that the $H_{a, b}\left(\operatorname{gr}_{v} \mathcal{L}^{-}, \operatorname{gr}_{v} \partial_{U}\right) \neq 0$, where $(a, b)$ is the bi-grading introduced in 4.1.11 Then $(a, b)=(-2 h(v)-2|K|,|K|)$ for some $K$.

Proof. Define $\psi: \operatorname{gr}_{v} \mathcal{L}^{-} \rightarrow \mathcal{E}[U]$ by $\psi\left(U^{m} \square(v, K)\right)=U^{m} z_{K}$. One verifies that it is an isomorphism, and $\partial_{U} \circ \psi=\psi \circ \mathrm{gr}_{v} \partial_{U}$. Hence induces an isomorphism at homological level too. By Theorem 5.2.8 (3) for the generators we can assume that $m=0$ and $\rho_{v}(K)=|K|$. Then the homological degree of $\square(v, K)$ is deg $=-2 h\left(v+e_{K}\right)+|K|=-2 h(v)-2 \rho_{v}(K)+|K|=$ $-2 h(v)-|K|$, while the degree of $z_{K}$ is $|K|$. For (b) note that the bi-degree of such $\square(v, K)$ is $(-2 h(v)-2|K|,|K|)$.

5.4. Proof of Theorem 4.2.1. Assume $v \notin \mathcal{S}$ and fix $i \in K_{0}$ such that $h(v)=h\left(v+e_{i}\right)$ (cf. Lemma 3.5.1), hence $h\left(v+e_{K}\right)=h\left(v+e_{K}+e_{i}\right)$ for any $K$ with $K \not \supset i$ by 3.5.2, Let $\phi: \operatorname{gr}_{v} \mathcal{L}^{-} \rightarrow \operatorname{gr}_{v} \mathcal{L}^{-}$be defined by

$$
\phi(\square(v, K))= \begin{cases}\square\left(v, K \cup i_{0}\right) & \text { if } i_{0} \notin K, \\ 0 & \text { if } i_{0} \in K .\end{cases}
$$

Then $\phi$ realizes a homotopy between the identity and the zero map: $\partial_{U} \phi+\phi \partial_{U}=i d$ on $\operatorname{gr}_{v} \mathcal{L}^{-}$. Hence $H_{*}\left(\operatorname{gr}_{v} \mathcal{L}^{-}\right)=0$. On the other hand, $\mathcal{H}(v)=\emptyset$, hence $H^{*}(\mathbb{P} \mathcal{H}(v))=0$ too.

If $v \in \mathcal{S}$ then parts (2) and (3) follow from Theorems 5.3.1 and 5.2.8(2). A possible second proof of part (1) is the following (for the first proof see 4.2):

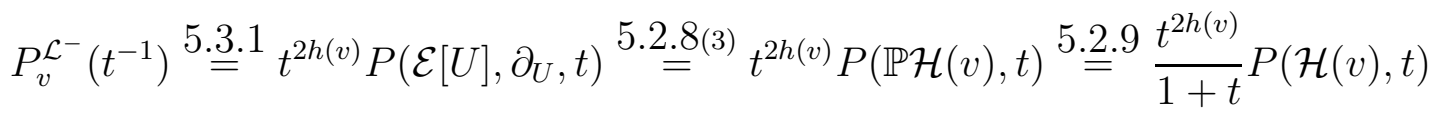

$$
\begin{aligned}
& \underline{5.2 .2} \frac{t^{2 h(v)}}{1+t} \cdot \sum_{K \subset K_{0}}(-1)^{|K|}(-t)^{\rho_{v}(K)}=\frac{(-t)^{h(v)}}{1+t} \cdot \sum_{K \subset K_{0}}(-1)^{|K|}(-t)^{h\left(v+e_{K}\right)} \text {. }
\end{aligned}
$$

\section{HeEgaARd Floer LiNK homology FOR ALGEBRAIC LiNKS}

6.1. We can now apply the results of the previous sections to the computation of the Heegaard Floer homology of algebraic links using the following result.

Theorem 6.1.1. ([11]) All algebraic links are L-space links.

Proposition 6.1.2. If $L$ is an algebraic link then its $H F L$-weight function coincides with the Hilbert function $h(v)$ up to an additive constant.

Proof. By Theorem 3.3.1 the Poincaré series coincides with the Alexander polynomial, hence with the Euler characteristic of $H F L^{-}$. The statement now follows from Theorems 3.4 .3 and 2.2 .11 
Theorem 6.1.3. Let $L$ be an algebraic $L$-space link corresponding to a plane curve singularity $C$. Then the spectral sequence defined in Theorem 2.2.10 collapses at $E^{2}$ page for all $v$ :

$$
\left.\mathrm{HFL}^{-}(L, v) \simeq \mathrm{HL}^{-}(L, v)=H_{*}\left(\operatorname{gr}_{v} \mathcal{L}_{C}^{-}\right) \quad \text { (as graded } \mathbb{Z} \text { modules }\right) .
$$

Proof. By Proposition 6.1.2 the $H F L$-weight function for $L$ coincides with the Hilbert function of $C$. Consider the spectral sequence of Theorem 2.2.10, Its $E^{2}$ page coincides with $H_{*}\left(\operatorname{gr} \mathcal{L}^{-}\right)$. We consider the bi-grading on $H_{*}\left(\operatorname{gr}_{v} \mathcal{L}^{-}\right)$, cf. 4.1.11, and we use the notations of the proof of Theorem 2.2.10. Note that the bi-grading $(a, b)$ coincides exactly with $(\nu,|K|)$. Hence, by Theorems 5.3.1 on the $E^{2}$ page all the non-trivial entries are on the line $\nu+2|K|+2 h(v)=0$, while the differential $d_{k}$ has bi-degree $(k-1,-k)$, hence two elements of this line are never connected by $d_{k}$ whenever $k \geq 2$. Hence $d_{k}=0$.

Remark 6.1.4. A similar spectral sequence was defined in the context of the subspace arrangements by Jewell [13], who also proved its degeneration at $E^{2}$ page.

Corollary 6.1.5. By Corollary 4.2.4 the set of $v$ such that $\mathrm{HFL}^{-}(L, v) \neq 0$ coincides with the semigroup of $C$. In particular, the support of $\mathrm{HFL}^{-}$determines the topological type of the algebraic link completely.

It is well known [27] that for $L$-space knots (hence for all algebraic knots) the dimension of the Heegaard Floer homology with given Alexander grading is at most 1. For algebraic links we get the following generalization of this result (it was independently proven in [14, Theorem 1.15] for general $L$-space links).

Corollary 6.1.6. If $L$ is an algebraic link, then $\operatorname{rank} \operatorname{HFL}^{-}(L, v) \leq 2^{r-1}$ for all $v \in \mathbb{Z}^{r}$. For $v$ large enough $\left(v \succeq l\right.$ in the notations of section 3.3) $\mathrm{rank}_{\mathrm{HFL}}^{-}(L, v)=2^{r-1}$.

Proof. It is clear from Theorem 5.2.8 that the total dimension of the homology of the complement to $r$ hyperplanes cannot exceed $2^{r}$ and equals $2^{r}$ if and only if the hyperplanes are independent. By the same theorem, projectivization of the arrangement halves the total dimension of its homology. It remains to note that by (3.3.3) the hyperplanes in the local arrangement $\mathcal{H}(v)$ are independent for $v \succeq l$.

\section{EXAMPLE. The HoPf LINK}

7.1. We illustrate the main results of the paper for the positive Hopf link, the link of the $A_{1}$ singularity $\{x y=0\}$. Its Alexander polynomial equals $\Delta\left(t_{1}, t_{2}\right)=1$.

A. Hyperplane arrangements. Let us describe the spaces $\mathcal{H}(v)$ explicitly. A function $g \in$ $\mathbb{C}[x, y]$ has order 0 on one of the components if and only its constant term is nonzero, and hence its order on the second component also equals 0 . Therefore

$$
\mathcal{H}(0,0)=\{\alpha+\text { higher order terms } \mid \alpha \neq 0\} \sim \mathbb{C}^{*}, \quad \mathcal{H}(a, 0)=\mathcal{H}(0, a)=\emptyset \text { for } a>0 .
$$

Furthermore, for $a, b>0$ the space $\mathcal{H}(a, b)$ is

$$
\left\{\alpha x^{b}+\beta y^{a}+\text { terms of type } \gamma x^{i} y^{j} \text { with }(i, j) \geq(1,1) \text {, or }(b+1,0) \text {, or }(0, a+1) \mid \alpha, \beta \neq 0\right\},
$$

Therefore $\mathcal{H}(a, b) \sim\left(\mathbb{C}^{*}\right)^{2}$, and

$$
H^{*}(\mathbb{P} \mathcal{H}(a, b))= \begin{cases}H^{*}(\text { point })=\mathbb{Z} & \text { if } a=b=0 \\ 0 & \text { if } a b=0,(a, b) \neq(0,0) \\ H^{*}\left(\mathbb{C}^{*}\right)=\mathbb{Z} \oplus \mathbb{Z} & \text { if } a, b>0\end{cases}
$$




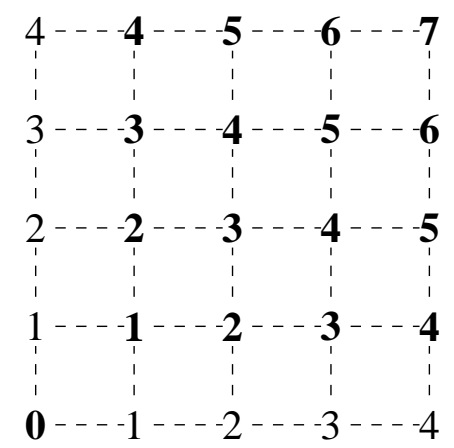

FIGURE 3. Values of the Hilbert function for $A_{1}$ singularity

Note that for $a, b>0$ the Euler characteristic of $\mathbb{P} \mathcal{H}(a, b))$ vanishes, so

$$
\left.\sum_{a, b \in \mathbb{Z}^{2}} t_{1}^{a} t_{2}^{b} \chi(\mathbb{P H}(a, b))\right)=1=\Delta\left(t_{1}, t_{2}\right)
$$

B. Local lattice homology. The Hilbert function of the $A_{1}$ singularity is (cf. Example 3.5.4):

$$
h(a, b)= \begin{cases}\max (a, b), & \text { if } \min (a, b)=0 \\ a+b-1, & \text { otherwise }\end{cases}
$$

It is shown in Figure 3. Let us compute the local lattice homology with the weight $h(v)$. For all $v=(a, b)$ the local lattice complex has 4 generators $a_{v}, \alpha_{v}, \beta_{v}, \Gamma_{v}$ over $\mathbb{Z}[U]$. Here $a_{v}$ can be identified with the point $v, \alpha_{v}$ and $\beta_{v}$ can be identified with the east- and northward pointing segments starting at $v$ and $\Gamma_{v}$ can be identified with the square with minimal vertex $v$. The differential is given by the equation:

$$
\begin{gathered}
\partial\left(a_{v}\right)=0, \partial\left(\alpha_{v}\right)=U^{h(a+1, b)-h(a, b)} a_{v}, \partial\left(\beta_{v}\right)=U^{h(a, b+1)-h(a, b)} a_{v}, \\
\partial\left(\Gamma_{v}\right)=U^{h(a+1, b+1)-h(a+1, b)} \alpha_{v}-U^{h(a+1, b+1)-h(a, b+1)} \beta_{v} .
\end{gathered}
$$

For $v=(0,0)$ one has $\partial\left(\alpha_{v}\right)=\partial\left(\beta_{v}\right)=U a_{v}, \partial\left(\Gamma_{v}\right)=\alpha_{v}-\beta_{v}$, so the homology is spanned by $a_{v}$. For $v=(a, 0), a>0$ one has $\partial\left(\alpha_{v}\right)=U a_{v}, \partial\left(\beta_{v}\right)=a_{v}, \partial\left(\Gamma_{v}\right)=\alpha_{v}-U \beta_{v}$, and the homology vanishes (similarly as for $v=(0, a)$ ). Finally, for $v=(a, b), a, b>0$ one has $\partial\left(\alpha_{v}\right)=\partial(\beta)=U a_{v}, \partial\left(\Gamma_{v}\right)=U\left(\alpha_{v}-\beta_{v}\right)$ and the homology is spanned by $a_{v}$ and $\alpha_{v}-\beta_{v}$, in agreement with (7.1.1). The homological degrees are $-2(a+b)+2$ and $-2(a+b)+1$. Note that $U$ acts by 0 on the homology in all cases.

C. Link Floer homology. Similarly to [29, Section 12], one can check that the minimal Heegaard Floer complex $C F L^{-}$has four $\mathbb{Z}\left[U_{1}, U_{2}\right]$-generators $\alpha, \beta, \gamma, \delta$ of Alexander gradings $(0,0),(1,0),(0,1),(1,1)$ and homological degrees $0,-1,-1,-2$. The differential is $\mathbb{Z}\left[U_{1}, U_{2}\right]$-linear given by the formula:

$$
d(\beta)=U_{1} \alpha+\delta, d(\gamma)=U_{2} \alpha+\delta, d(\alpha)=d(\delta)=0 .
$$

The filtered subcomplex $A^{-}(v)$ is spanned by all elements of Alexander grading greater than or equal to $v$. By definition, $\mathrm{HFL}^{-}(v)$ is the homology of the associated graded complexe gr $A^{-}(v)$. For $v=(0,0)$ the complex gr $A^{-}(0,0)$ is generated over $\mathbb{Z}$ by a single element $\alpha$. For $a>0$ the complex gr $A^{-}(a, 0)$ is generated over $\mathbb{Z}$ by $U_{1}^{a} \alpha, U_{1}^{a-1} \beta$, with the differential

$$
d_{\mathrm{gr}}\left(U_{1}^{a-1} \beta\right)=U_{1}^{a} \alpha \text {. }
$$


Therefore $\operatorname{gr} A^{-}(a, 0)$ (and similarly gr $\left.A^{-}(0, a)\right)$ is acyclic. Finally, for $a, b>0$ the complex gr $A^{-}(a, b)$ is generated by $U_{1}^{a} U_{2}^{b} \alpha, U_{1}^{a-1} U_{2}^{b} \beta, U_{1}^{a} U_{2}^{b-1} \gamma$ and $U_{1}^{a-1} U_{2}^{b-1} \delta$, with the differential

$$
d_{\mathrm{gr}}\left(U_{1}^{a-1} U_{2}^{b} \beta\right)=d_{\mathrm{gr}}\left(U_{1}^{a} U_{2}^{b-1} \gamma\right)=U_{1}^{a} U_{2}^{b} \alpha .
$$

Its homology (in agreement with (7.1.1) ) equals

$$
\operatorname{HFL}^{-}(a, b)=H^{*}\left(\operatorname{gr} A^{-}(a, b)\right) \simeq \mathbb{Z}\left\langle U_{1}^{a-1} U_{2}^{b-1} \delta, U_{1}^{a-1} U_{2}^{b} \beta-U_{1}^{a} U_{2}^{b-1} \gamma\right\rangle .
$$

D. Filtered subcomplexes. Let us also compute the homology of $A^{-}(v)$ for various $v$. The complex $A^{-}(0,0)$ coincides with $C F L^{-}$and its homology has the form

$$
H^{*}\left(A^{-}(0,0)\right)=\mathbb{Z}\left[U_{1}, U_{2}\right]\langle\alpha\rangle /\left(U_{1} \alpha=U_{2} \alpha\right) \simeq \mathbb{Z}[U]\langle\alpha\rangle .
$$

For $a>0$ the complex $A^{-}(a, 0)$ is generated over $\mathbb{Z}\left[U_{1}, U_{2}\right]$ by $U_{1}^{a} \alpha, U_{1}^{a-1} \beta, U_{1}^{a} \gamma$ and $U_{1}^{a-1} \delta$. One can check that

$$
H^{*}\left(A^{-}(a, 0)\right) \simeq \mathbb{Z}[U]\left\langle U_{1}^{a-1} \delta\right\rangle,
$$

and its generator has homological degree $-2 a$. Similarly, $H^{*}\left(A^{-}(0, b)\right) \simeq \mathbb{Z}[U]\left\langle U_{2}^{b-1} \delta\right\rangle$ generated at degree $-2 b$. Finally, for $a, b>0$ the subcomplex $A^{-}(a, b)$ is generated over $\mathbb{Z}\left[U_{1}, U_{2}\right]$ by $U_{1}^{a} U_{2}^{b} \alpha, U_{1}^{a-1} U_{2}^{b} \beta, U_{1}^{a} U_{2}^{b-1} \gamma$ and $U_{1}^{a-1} U_{2}^{b-1} \delta$. One can check that

$$
H^{*}\left(A^{-}(a, b)\right) \simeq \mathbb{Z}[U]\left\langle U_{1}^{a-1} U_{2}^{b-1} \delta\right\rangle,
$$

and its generator has homological degree $-2 a-2 b+2$. Therefore for all $v$ the subcomplex $A^{-}(v)$ is a free $\mathbb{Z}[U]$-module of rank 1 , and its generator has homological degree $-2 h(v)$.

\section{REFERENCES}

[1] V. I. Arnold, S. M. Gusein-Zade, A. N. Varchenko. Singularities of differentiable maps. Vol. 2, Birkhäuser, 1985.

[2] M. Borodzik, C. Livingston. Semigroups, $d$-invariants and deformations of cuspidal singular points of plane curves. arXiv:1305.2868

[3] E. Brieskorn. Sur les groupes de tresses. Seminaire Bourbaki 1971/72. Lecture Notes in Math. 317, Springer, 1973, 21-44.

[4] A. Campillo, F. Delgado, S. M. Gusein-Zade. The Alexander polynomial of a plane curve singularity via the ring of functions on it. Duke Math J. 117 (2003), no. 1, 125-156.

[5] A. Campillo, F. Delgado, S. M. Gusein-Zade. Integrals with respect to the Euler characteristic over spaces of functions and the Alexander polynomial. Proc. Steklov Inst. Math. 238 (2002), no. 3, 134-147.

[6] A. Campillo, F. Delgado, S. M. Gusein-Zade. Multi-index filtrations and motivic Poincaré series. Monatshefte für Mathematik. 150 (2007), no.3, 193-210.

[7] A. Campillo, F. Delgado, K. Kiyek. Gorenstein property and symmetry for one-dimensional local CohenMacaulay rings. Manuscripta Math. 83 (1994), no. 3-4, 405-423.

[8] D. Cohen, G. Denham, M. Falk, H. Schenck, A. Suciu, H. Terao, S. Yuzvinsky. Complex Arrangements: Algebra, Geometry, Topology. Draft of September 4, 2009.

[9] D. Eisenbud, W. Neumann. Three-dimensional link theory and invariants of plane curve singularities. Annals of Mathematics Studies, 110. Princeton University Press, Princeton, NJ, 1985.

[10] E. Gorsky. Combinatorial computation of the motivic Poincaré series. J. Singul. 3 (2011), 48-82.

[11] E. Gorsky, A. Némethi. Links of plane curve singularities are $L$-space links. arXiv:1403.3143.

[12] M. Hedden. On knot Floer homology and cabling. II. Int. Math. Res. Not. IMRN 2009, no. 12, 2248-2274,

[13] K. Jewell. Complements of sphere and subspace arrangements. Topology Appl. 56 (1994), no. 3, $199-214$.

[14] Y. Liu. L-space surgeries on links. arXiv:1409.0075

[15] C. Manolescu, P. Ozsváth. Heegaard Floer homology and integer surgeries on links. arXiv:1011.1317

[16] J. Milnor. Singular points of complex hypersurfaces, Annals of Math. Studies, 61, Princeton University Press, 1968.

[17] J.J. Moyano-Fernández. Poincaré series for plane curve singularities and their behaviour under projections. J. Pure Appl. Algebra 219 (2015), no. 6, 2449-2462. 
[18] J.J. Moyano-Fernández, W. A. Zúñiga-Galindo. Motivic Zeta Functions for Curve Singularities. Nagoya Math. J. 198 (2010), 47-75.

[19] A. Némethi. The Seiberg-Witten invariants of negative definite plumbed 3-manifolds. J. Eur. Math. Soc. (JEMS) 13 (2011), no. 4, 959-974.

[20] A. Némethi. Poincaré series associated with surface singularities. Singularities I, 271-297, Contemp. Math., 474, Amer. Math. Soc., Providence, RI, 2008.

[21] A. Némethi. The cohomology of line bundles of splice-quotient singularities. Adv. Math. 229 (2012), no. 4, $2503-2524$.

[22] A. Némethi. Two exact sequences for lattice cohomology. Noncommutative geometry and global analysis, 249-269, Contemp. Math., 546, Amer. Math. Soc., Providence, RI, 2011.

[23] A. Némethi. Lattice cohomology of normal surface singularities. Publ. Res. Inst. Math. Sci. 44 (2008), no. 2, 507-543.

[24] P. Orlik and L. Solomon. Combinatorics and topology of complements of hyperplanes. Invent. Math. 56 (1980), 167-189.

[25] P. Orlik, H. Terao. Arrangements of Hyperplanes. Springer, 1992.

[26] P. Ozsváth, Z. Szabó. On the Floer homology of plumbed three-manifolds. Geometry and Topology 7 (2003), 185-224.

[27] P. Ozsváth, Z. Szabó. On knot Floer homology and lens space surgeries. Topology 44 (2005), no. 6, $1281-$ 1300.

[28] P. Ozsváth, Z. Szabó. Holomorphic discs and knot invariants. Adv. Math., 186 (1), 2004, 58-116.

[29] P. Ozsváth, Z. Szabó. Holomorphic disks, link invariants and the multi-variable Alexander polynomial. Algebr. Geom. Topol. 8 (2008), no. 2, 615-692.

[30] P. Ozsváth, Z. Szabó. Holomorphic discs and topological invariants for closed three-manifolds. Ann. of Math. (2) 159 (2004), no. 3, 1027-1158.

[31] P. Ozsváth, Z. Szabó, A. Stipsicz. A spectral sequence on lattice homology.arXiv:1206.1654

[32] P. Ozsváth, Z. Szabó, A. Stipsicz. Knot lattice homology in L-spaces. arXiv:1207.3889.

[33] J. Rasmussen. Knot polynomials and knot homologies, in Geometry and Topology of Manifolds, Boden et. al. eds., Fields Institute Communications 47 (2005), 261-280.

[34] R. P. Stanley. An introduction to hyperplane arrangements. Geometric combinatorics, 389-496, IAS/Park City Math. Ser., 13, Amer. Math. Soc., Providence, RI, 2007.

[35] G. Torres. On the Alexander polynomial. Ann. of Math. 57 (1953), no.1, 57-89.

[36] M Yamamoto. Classification of isolated algebraic singularities by their Alexander polynomials. Topology $\mathbf{2 3}$ (3) (1984), 277-287.

Eugene Gorsky, Mathematics Department, Columbia University, 2990 Broadway, New York, NY 10027 USA

Department of Mathematics, UC Davis, One Shields Ave, Davis, CA 95616

International Laboratory of Representation Theory and Mathematical Physics, NRUHSE, 7 VAVIlova St., Moscow, Russia 117312

E-mail address: egorsky@math.columbia.edu

András Némethi, Alfréd Rényi Institute of Mathematics, Hungarian ACademy of SciENCES, REÁltanOda utCa 13-15, H-1053, BudAPest, Hungary

E-mail address: nemethi.andraserenyi.mta.hu 